\title{
Gecko-Like Dry Adhesive Surfaces and Their Applications: A Review
}

\author{
Wei Wang ${ }^{1} \cdot$ Yang $\mathrm{Liu}^{1} \cdot$ Zongwu Xie $^{1}$
}

Received: 2 June 2021 / Revised: 9 August 2021 / Accepted: 15 August 2021 / Published online: 6 October 2021

(c) The Author(s) 2021

\begin{abstract}
Gecko has the ability to climb flexibly on various natural surfaces because of its fine layered adhesion system of foot, which has motivated researchers to carry out a lot of researches on it. Significant progresses have been made in the gecko-like dry adhesive surfaces in the past 2 decades, such as the mechanical measurement of adhesive characteristics, the theoretical modeling of adhesive mechanism and the production of synthetic dry adhesive surfaces. Relevant application researches have been carried out as well. This paper focuses on the investigations made in recent years on the gecko-like dry adhesive surfaces, so as to lay the foundation for further research breakthroughs. First, the adhesion system of gecko's foot and its excellent adhesive characteristics are reviewed, and the adhesive models describing the gecko adhesion are summarily reviewed according to the different contact modes. Then, some gecko-like dry adhesive surfaces with outstanding adhesive characteristics are presented. Next, some application researches based on the gecko-like dry adhesive surfaces are introduced. Finally, the full text is summarized and the problems to be solved on the gecko-like dry adhesive surfaces are prospected.
\end{abstract}

Keywords Gecko adhesion $\cdot$ Gecko-like dry adhesive surfaces $\cdot$ Climbing robot $\cdot$ Robot gripper $\cdot$ Medical adhesive tape

\section{Introduction}

The surface of the gecko's foot is the most representative bio-adhesive surface in the biological world. Its unique micro-system structure and outstanding adhesive characteristics inspire researches and designs of new type of biomimetic dry adhesive surfaces. Since the gecko's extraordinary adhesive capacity for the surface was discovered, extensive research on it has been carried out [1-3]. As shown in Fig. 1, human have made various investigations to uncover the mystery behind the gecko's adhesive system and use this as a reference to invent the gecko-like dry adhesive surfaces, so as to replicate the excellent adhesive characteristics of gecko's foot and apply the dry adhesive surfaces to all areas of human life and production. Although researchers have invented many gecko-like dry adhesive surfaces based on the inspiration from the adhesive system of gecko's foot, and have had a deeper understanding on some important factors that affect their adhesive performance and characteristics, there are still some unresolved problems and challenges. To

Zongwu Xie

xiezongwu@hit.edu.cn

1 State Key Laboratory of Robotics and System, Harbin Institute of Technology, Harbin 150001, China further promote the adhesive capacity of the corresponding gecko-like dry adhesive surfaces, the more complete adhesive model coupling the adhesion and the friction is needed to explore more effective strategies to optimize the microstructures on the dry adhesive surface. Moreover, the multi-scale hierarchical microstructure array more similar to the gecko's foot is required to significantly improve the surface adaptability of the gecko-like dry adhesive surfaces. In particular, the corresponding manufacture technology also needs to be broken through. In addition, the related application research based on the existing gecko-like dry adhesive surfaces also needs to be further followed up, and the shortcomings in practical application will further promote the improvement and optimization of gecko-like dry adhesive surface.

In this article, the important progresses in the research of gecko-like dry adhesive surfaces in recent years are systematically reviewed mainly from three aspects: measuring and explaining the gecko adhesion, designing and manufacturing the gecko-like dry adhesive surfaces, exploring the corresponding applications based on gecko-like dry adhesive surfaces. The purpose of this review is to summarize the current research progresses of gecko-like dry adhesive surfaces, and to lay a foundation for further promoting the research of gecko-like dry adhesive surfaces. 


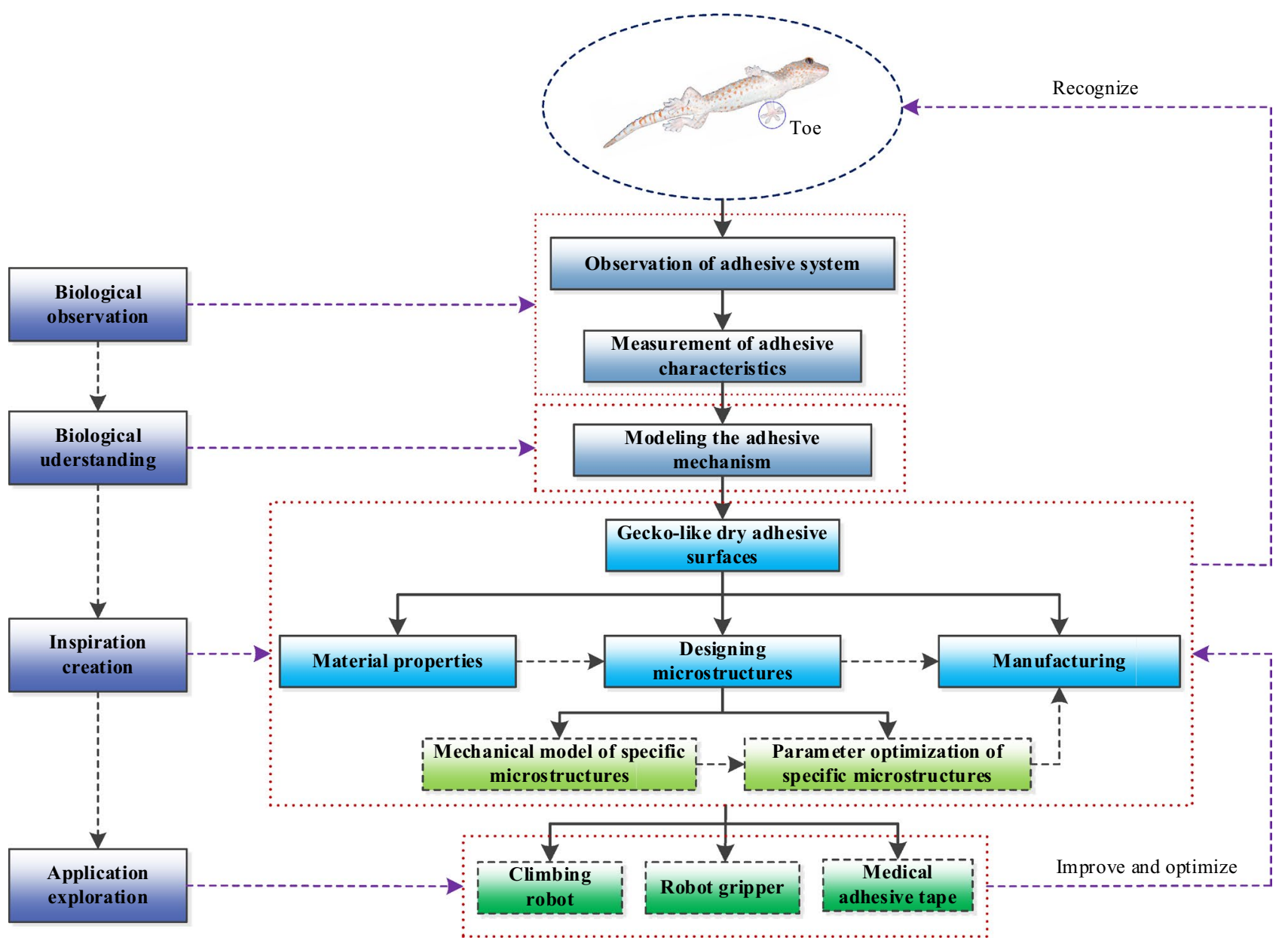

Fig. 1 The schematic diagram of the research process of gecko adhesion

\section{The Gecko Adhesion}

\subsection{The Structure of the Gecko's Adhesive System}

Human cognition for the gecko's adhesive system is gradually improved with the development of related observation and measurement technology. The earliest research records on the adhesive capacity of gecko can be traced back to Aristotle's Historia Animalium in the fourth century BC, which records the macroscopic movement of gecko climbing up and down on trees at will [1]. However, due to the limitation of observation technology, researchers did not have a clear understanding on the microstructure of the gecko's adhesive system in the following 2000 years. It was not until the invention of the electron microscope in the 1950s, researchers observed that the gecko's adhesive system is composed of micro-nano multi-scale layered setae [4]. Figure 2 shows the layered microstructure of adhesive system of Tokay gecko, and the specific size characteristics are shown in Table 1. The gecko's setae are mainly composed of $\beta$ keratin with a modulus of about 1.6 GPa, and the hard setae are helpful for the self-cleaning and anti-entanglement of the adhesive system [5-7]. Due to the layered microstructure of setae, the equivalent elastic modulus of the adhesive system is only about $80-90 \mathrm{kPa}$, which greatly improves the surface adaptability of the adhesive system [8].

\subsection{The Source of Gecko Adhesion}

Researchers had put forward different hypotheses about the source of gecko adhesion, such as mucus adhesion, vacuum adhesion, electrostatic adhesion, and micro-interlock, but they were all denied by subsequent experimental observations, and the remaining most potential sources of adhesion were the Van der Waals force and the capillary force [9]. However, there had been no definite conclusion about the source of gecko adhesion for a long time due to the limitation of measurement technology in the past. Until the end of the twentieth century, the development of MEMS sensing technology brought dawn light to the fine measurement 


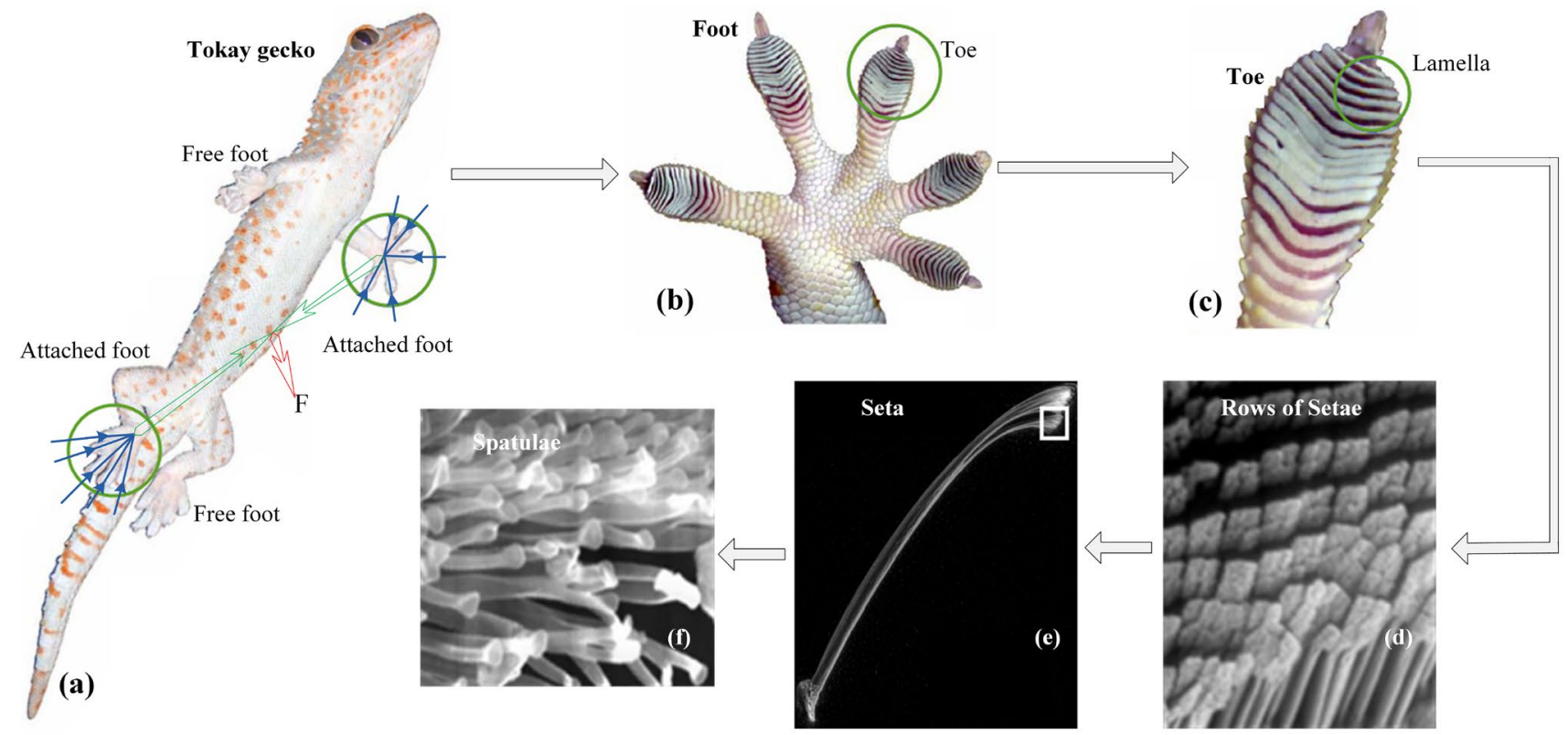

Fig. 2 The gecko's adhesive system. a-c The macroscopic compositions of gecko's adhesive system [50]; d-f The microscopic compositions of gecko's adhesive system [9]

Table 1 The size characteristics of adhesive system of Tokay Gecko $[4,5,174,175]$

\begin{tabular}{lllll}
\hline Scale & Structure & Size & Density & Adhesion \\
\hline Millimeter & Toe & Areas: $227.1 \mathrm{~mm}^{2} \pm 10.81 \mathrm{~mm}^{2}$ & 5 per foot & $20.04 \mathrm{~N} \pm 1.33 \mathrm{~N}$ \\
Micron & Lamella & Length: $400 \mu \mathrm{m}-600 \mu \mathrm{m}$ & 20 row per foot & - \\
& Seta & Diameter: $5 \mu \mathrm{m}-10 \mu \mathrm{m}$, length: & 14,000 setae $/ \mathrm{mm}^{2}$ & $194 \mu \mathrm{N} \pm 25 \mu \mathrm{N}$ \\
& & $30 \mu \mathrm{m}-130 \mu \mathrm{m}$ & \\
Nanometer & Spatula & Diameter: $100 \mathrm{~nm}-200 \mathrm{~nm}$, length: & $\begin{array}{c}100-1000 \text { spatulae/ } \\
\text { seta }\end{array}$ & $10 \mathrm{nN}$ \\
& \multicolumn{2}{c}{$\begin{array}{c}2-5 \mu \mathrm{m} \\
\end{array}$} & Spatula tip & Length: $500 \mathrm{~nm}$, width: \\
& $200 \mathrm{~nm}-300 \mathrm{~nm}$, thickness: $10 \mathrm{~nm}$ & & $10 \mathrm{nN}$ \\
\hline
\end{tabular}

of gecko adhesion, and laid a foundation for the extensive researches on the adhesive mechanism of gecko [10]. Originally, the adhesive measurement processes of a single gecko's seta based on two different micro-cantilever MEMS sensors correspond to two motion mode (parallel dragging and vertical peeling of seta), respectively. In the parallel dragging mode, the gecko's seta first contacts with the tip surface of the micro-cantilever sensor under a certain vertical preload, and then is dragged along the contact surface [11]. The forces generated by the gecko's seta in the process of vertical preloading and parallel dragging are sensed by two independent piezoresistive sensing units of the cantilever due to the orthogonal decoupling flexibility of the biaxial micro-cantilever in two directions. In the vertical peeling mode, the single gecko's seta is also first applied with a certain vertical preload to make it come into effective contact with the surface of wire sensor, and then it is peeled vertically away from the surface of the sensor. The force generated by the seta in the vertical peeling process is reflected by the deflection of the wire sensor, and the corresponding deflection monitored by the digital camera finally converted into the adhesion after being calibrated [11]. In view of the above accurate measurements of the adhesion of the single gecko's seta, it was found that the adhesion of the single seta during the parallel dragging and vertical peeling process is just within the ranges of the Van der Waals force predicted by the simple mathematical model, which provides indirect support for the hypothesis that the gecko adhesion is derived from the Van der Waals force [11]. Furthermore, the adhesive performances of the single gecko's toe and the single gecko's seta on the hydrophobic and hydrophilic surfaces were also explored following similar measurement principles. It was found that neither the tangential adhesion generated by the single gecko's toe nor the normal adhesion generated by the single gecko's seta does not have a significant difference on the hydrophobic and hydrophilic 
surfaces $[9,12]$. The consistency of the adhesive capacity of gecko's toe and seta on hydrophobic and hydrophilic surfaces is consistent with the fact that Van der Waals force is not affected by the polarity of surface, but is contrary to the fact that capillary force depends on the polarity of surface. Therefore, the above measurement results provide the most direct evidence for the hypothesis that the gecko adhesion originates from the Van der Waals force. Moreover, the study also found that the size of gecko's spatula predicted through the experimentally measured adhesion and the calculation model of Van der Waals force is similar to the actual size of gecko's spatula by the measurement. As a result, this further confirms that the above-mentioned measurement results are quantitatively consistent with the Van der Waals interaction between the gecko's seta and the substrate and it also shows that the smaller spatula will produce greater adhesion for a given adhesion energy [12]. In addition, the research also confirmed that the artificial gecko's spatula constructed based on two different materials is similar to the natural gecko's spatula in terms of the adhesive function [12]. Combining these and considering that the Van der Waals force is a ubiquitous weak intermolecular force and strongly depends on the intermolecular distance without being affected by the surface polarity, the gecko adhesion based on the interaction mechanism of Van der Waals should be more dependent on the geometry of spatula rather than its material properties or surface chemistry. The above inference can be also supported by the fact that some geckos and insects have developed the refined microscopic setae instead of the specific surface chemicals to produce effective adhesion that can adhere most surfaces.

Although the experimental measurements confirmed that the adhesion of gecko's foot is mainly derived from the Van der Waals force between the contact surfaces, it did not confirm whether the capillary force and humid environment have an effect on the adhesion of gecko's foot. In this case, some additional experimental investigations needed to be carried out. By peeling off the single gecko's spatula on the wafer with different humidity, it was found that low humidity is helpful to enhance the adhesive capacity of gecko's spatula in the nanometer scale. However, the Hamaker constant decreases when the humidity increases, so that the adhesion is reduced or even disappear [13]. The effect of humidity on the adhesive capacity of gecko's spatula is the result of change of the effective short-distance interaction caused by the monolayer water film between the spatula and the contact surface. Furthermore, it was found that the effect of relative humidity on the adhesion of gecko varies with the contact angle based on the adhesive model considering capillary force. For example, the adhesion increases with the increase of relative humidity when the contact angle is small, but it decreases with the increase of relative humidity when the contact angle is large [14]. Another study about the adhesive performance of gecko's seta in a relatively humid surface also found that the adhesion and friction of gecko spatula increase with the increase of relative humidity [15]. However, that study believed that this effect is not the result of capillary force, but that the increase in relative humidity softens the seta and increases its viscous damping, thereby increasing the adhesion.

So far, researchers basically agree that the Van der Waals force is still the sole source of gecko adhesion, and the capillary force and relative humidity have an enhanced effect on it.

\subsection{The Adhesive Characteristics of Gecko's Foot}

The adhesive system of gecko's foot is currently the most versatile and effective biological adhesive system known in nature. Its unique multi-scale layered setae based on the interaction of Van der Waals forces not only endow the gecko with the strong adhesion, but also give it with unique adhesive characteristics (as shown in Fig. 3). As a result, the gecko has the ability to easily and quickly climb on various surfaces. Studying the adhesive characteristics of gecko's foot is helpful to understand the physical mechanism of gecko's adhesive climbing, and provides ideas and guidance for the design of gecko-like dry adhesive surfaces. A series of detailed experimental tests have been also conducted to ascertain its various adhesive characteristics since the microscopic hierarchical structure of gecko's foot was clearly understood.

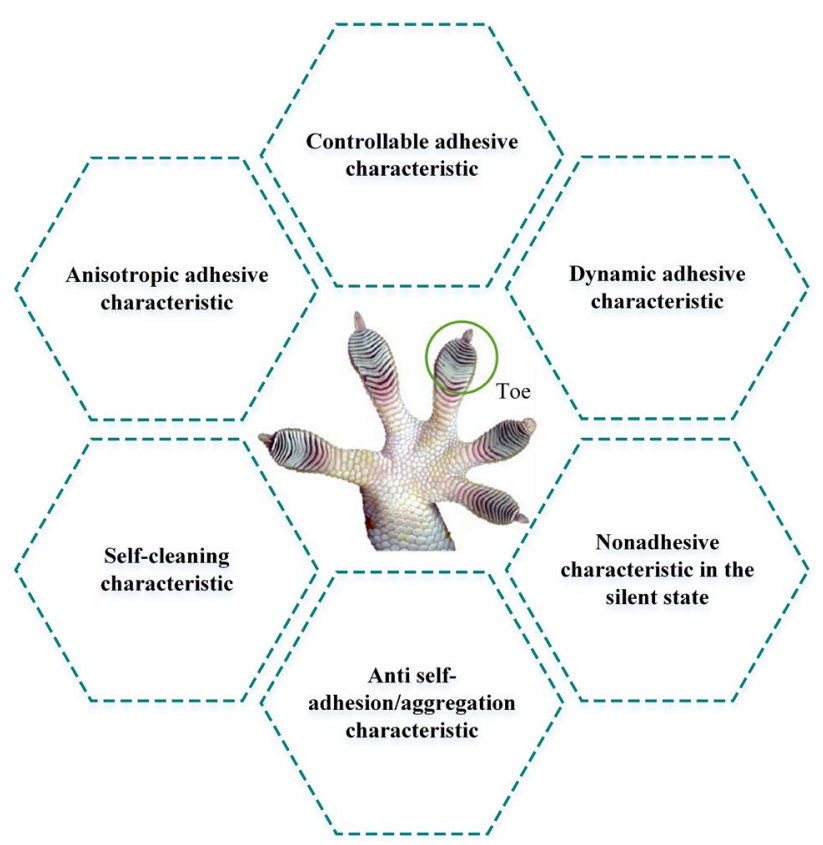

Fig. 3 The adhesive characteristics of gecko's foot 


\subsubsection{Anisotropic Adhesive Characteristic}

The anisotropic adhesive characteristic describes that the adhesion of gecko's seta changes with the direction of the seta, and shows differences in different directions. Experimental studies showed that the specific orientation of the seta can not only produce significant adhesion, but also minimize the force required to peel off the seta [11]. There is a critical angle of $30^{\circ}$ for the detachment of the seta: the required force for detachment is the largest when the angle between the spatula shaft and the contact surface meets the critical angle; when the angle is greater than the critical angle, the adhesion gradually decreases, resulting in the detachment of the seta from the surface $[9,11]$. The other study also confirmed that the adhesion of seta is highly sensitive to the angle between the seta and the contact surface (the peeling angle), and the adhesion of seta shows obvious anisotropy due to the change of the peeling angle. In particular, the tangential adhesion gradually decreases as the peeling angle increases $\left(0^{\circ}-25^{\circ}\right)[16]$.

\subsubsection{Controllable Adhesive Characteristic}

The controllable adhesive characteristic describes that gecko controls the adhesive state of its seta and even fine adjusts the adhesion of the seta in the specific direction. Gecko can switch between fast attachment and detachment within milliseconds, and generate almost zero reacting force in the process, that is, gecko only needs low preload for attachment and low peeling force for detachment, which is inseparable from the macro-control of millions of setae on the toes by the gecko. Preliminary test analysis suggested that it is related to the unique macroscopic movements of gecko's toes which contain uncurling toes for attachment and hyperextending toes for detachment $[9,17]$. However, the actual experimental measurements found that the normal adhesion of seta is linearly related to the tangential friction of seta, and the tangential friction can directly regulate the normal adhesion [18]. The regulation mechanism can be illustrated by the frictional adhesion model, which also provides an explanation for requiring low peeling force when detaching $[18,19]$. Simulating the attachment and detachment of the seta through the surface force measuring device also found that the normal adhesion and the tangential friction are coupled, and the coupling relationship shows a difference in the grasping and releasing directions of the seta due to their anisotropic adhesive characteristic, so gecko can control the adhesive state by switching the direction of seta [20]. Moreover, the natural bended and inclined geometry of the seta plays an important role in the switch control of the adhesive state [21]. Other studies also showed that the tangential friction of gecko is affected by the preload and tangential sliding distance, which suggests that the preload and tangential sliding distance may be able to indirectly regulate its normal adhesion by adjusting the tangential friction [22-24]. In addition, study also confirmed that gecko can also show obvious characteristic of frictional adhesion on its feet during movement through the complex and accurate synergy between the toes [25]. Therefore, gecko can precisely control the normal adhesion through the shear force on the macroscale, thereby allowing the attachment and detachment to be carried out with negligible force. In addition, the study also founded that gecko also use their body's compliance to control the force on their feet to avoid overloading the single contact area between their feet and the climbing surface [25].

\subsubsection{Dynamic Adhesive Characteristic}

The dynamic adhesive characteristic describes that the gecko can hold their adhesion during the sliding process. When the adhesive state of gecko changes from the static adhering to the dynamic sliding due to the external disturbances, the seta on gecko's toes can detach and reattach independently [26]. The gradual sliding can prompt the seta to re-establish adhesion uniformly, thereby preventing catastrophic suddenly detachment and fall. Among the above-mentioned tests on the adhesive characteristic of gecko's seta, the high adhesion maintained by the seta in the dragging process is the best evidence for its dynamic adhesive characteristic [9, 11, 17-19]. Based on the stick-slip model describing the dynamic frictional adhesion of seta, the friction and adhesion of seta increases with the increase of shear dragging speed at the beginning of sliding, and it is believed that the stable friction and adhesion held by the seta during the sliding process may be result of randomly stick-slip of a large number of single seta with high resonance frequency [27]. The dynamic response of gecko's seta during sliding is helpful to prevent sudden drop and recover from sliding.

\subsubsection{Self-Cleaning Characteristic}

The self-cleaning characteristic describes the ability that the setae of gecko can resist and self-clean external contaminated particles during the repeated adhesive process, which effectively improves the durability of the setae. Experiments showed that contaminated particles (silica-alumina ceramic microspheres) can significantly reduce the adhesive performance of setae/toes, but the contaminated particles on the setae can be reduced after an average of eight cycles of adhesion detachment, and the adhesive capacity can be restored $35.6 \%$, which is sufficient to support the overall weight of the gecko [28]. The fact that the gecko restores the clean state and adhesive capacity of the contaminated toes through repeated attachment and detachment proves that its toes have the self-cleaning characteristic, and the self-cleaning method through repeated contact is attributed to the imbalance of 
the adhesion of the spatula and the substrate on the particle, as well as the self-cleaning effect depends on the size of the spatula and particles. Considering that the actual movement of gecko's foot is accompanied by the digital hyperextension of toe (curling the toe from the end to the front), the selfcleaning behavior of gecko's toe on the macro-scale was also explored to find out whether the digital hyperextension of toe has an effect its self-cleaning effect. The results showed that the gecko with the digital hyperextension of toe has a better self-cleaning effect, that is, the gecko uses only four walking steps to restore its corresponding adhesion by nearly $80 \%$, which is twice the self-cleaning effect of gecko without the digital hyperextension of toe [29]. The digital hyperextension of gecko's toe plays an important role in improving its self-cleaning ability, and the role is due to the dynamic jump-off of seta stimulated by the digital hyperextension of toe. The reason why the dynamic jump-off of seta can produce the self-cleaning effect is that there is the difference in response to the normal peeling speed between the adhesion of the substrate to the particles and the adhesion of the seta to the particles [30]. The former shows an increasing trend with the increase of the normal peeling speed, while the latter has almost no dependence on the normal peeling speed. Besides, the normal peeling speed generated by the dynamic jump-off of gecko seta is sufficient to make it always show self-cleaning characteristic during exercise [30]. In addition, the setae with multi-level layered microstructures are proved to be super-hydrophobic in the non-adhesive state, so they should also be able to clean the surface through the rolling of water droplets as the surface of a lotus leaf $[12,31]$.

\subsubsection{Non-adhesive Characteristic in the Silent State}

The non-adhesive characteristic in the silent state describes the characteristic that the gecko's setae do not exhibit the adhesion in the silent state. By measuring the contact angle of water droplet on the surface of isolated setae array, it was found that the super-hydrophobic setae array is in the nonadhesive state when they are silent, and it can be inferred that the non-adhesive state is derived from the fact that there is very little contact between the undeformed setae and the interface based on the Cassie's law [31]. The results from the adhesion measurements of isolated seta can also indirectly prove this characteristic, that is, the adhesive system of gecko requires a certain external stimulus (vertical preload and horizontal dragging displacement) to show the adhesive capacity [11]. The non-adhesive characteristic in the silent state can improve the anti-pollution ability of setae on the one hand, and on the other hand, it also helps the gecko to actively choose the adhesive time and position, thereby helping it to climb quickly on various surfaces.

\subsubsection{Anti-self-adhesion/Aggregation Characteristic}

The anti-self-adhesion/aggregation characteristic describes the ability of gecko's setae to resist self-adhesion or entanglement between setae. The slender geometry and layered structure of the gecko's setae make it have good flexibility, but the densely arranged setae do not show entanglement or aggregation. The unique anti-self-adhesion or aggregation characteristic still endows the setae with good adhesive performance while ensuring that the setae adapt to the surface, which may be attributed to the fact that the adhesion between the surfaces of setae is equivalent to the elastic restoring force inside setae [32].

\subsection{The Adhesive Model of Gecko's Foot}

Building the adhesive model of gecko's foot cannot only help explain its specific adhesive characteristics, but also provide insight into its related parameters, and then provide guidance for the design and optimization of the gecko-like dry adhesive surfaces.

At present, many mechanical models describing the interface adhesion have been constructed to explain the adhesive mechanism of gecko's foot [1-3]. The end of the fiber on the gecko's foot is a nanometer scale spatula-like microstructure which is the most direct utility unit in the adhesive process of gecko's foot, so some corresponding adhesive models were constructed in the form of tip contact based on the classical contact models and the fracture mechanics [33-42]. In these adhesive models constructed in the form of tip contact, the single microstructure is usually regarded as a simple cylinder or cantilever beam. Based on the researches of these simple models, some useful conclusions could be obtained. In these adhesive models based on the cylindrical fiber hypothesis, the researchers found that the bucking effect of fiber controlled by the ratio of length-to-diameter of fiber contributes to the initial uniform contact between the fiber and the rough interface [33]; the tip shape of fiber has an effect on the adhesive capacity, especially the fiber with a flat tip can show stronger adhesion [37]; and the enhanced adhesion can be achieved through the contact refinement of the fiber structure [34], but the adhesion will eventually gradually approach the theoretical strength of the Van der Waals force [37]. In view of these adhesive models that fiber is regarded as the simple cantilever, researchers discovered that the inclination of the fiber helps it adapt to the contact surface, and the orientation of fiber has an effect on the peeling of the fibers, as well as changing the tangential load can achieve rapid release of the fibers to a certain extent $[35,36$, 41]. In addition, to integrate the hierarchical flexibility of the fiber into the adhesive model, some researchers characterized the flexibility of the adhesive system by the spring. On the basis of these adhesive models containing different 
levels of springs, it was found that the flexibility of the fiber helps itself to adhere on rough surfaces, and the multi-level layered structure has better adaptability and adhesive capacity $[39,40,42]$.

Although the simple analytical adhesive models mentioned above can provide much guidance for understanding the gecko-like dry adhesive surfaces, these models are not so accurate in the characterization of the interface adhesion of the actual fiber due to the excessive simplification of the models in terms of the structure and material property of fiber. Some researchers also tried to establish numerical models for adhesion of fiber. For example, Gao et al. studied the adhesive strength of fiber with different tips and explored the influence of contact size on their adhesive strength under the framework of numerical simulation including the precise tip geometry and deformation of fibers, which laid the foundation for the later development of the dry adhesive surface based on the mushroomshaped microstructures [43-45]. At the same time, Gao et al. also built a finite-element model in which an isolated fiber is in contact with the rigid substrate [37]. It was found that the fiber has a critical angle of 30 degrees, which is consistent with the actual measurement result of gecko's seta, and the model also directly showed that the asymmetric design of the seta is especially beneficial for the fast switching between attachment and detachment.

The detachment process of gecko's fiber is very similar to the peeling process of tape, which has greatly motivated researchers to study the adhesive model in the form of side contact based on the model of film peeling [46-48]. Among them, the most classic model is undoubtedly the peeling model of tape constructed by Tian et. al [49]. In this model, the adhesion and friction between the spatula and the substrate are taken into account, but issues such as the shape of the peeling area and the possible nonlinear deformation of fiber are not taken into consideration. Therefore, some improved works have been carried out. For instance, Pesika et al. established a model considering the geometry of the peeling area of an isolated spatula [50]; Chen et al. constructed a generalized peeling model of Kendall involving the pre-stretching of fiber [51]; Peng et al. considered the relatively real finite adhesive length, and constructed a simulation model of plane strain describing the peeling of spatula based on the cohesive element, as well as discussed the influence of non-uniform pretension on the adhesive characteristics of nanometer film and layered adhesive structure [52]; Cheng et al. also created a plane strain model of peeling film considering that gecko can generate nonuniform pretension in spatula by the tangential slip, so as to explore the effect of the tangential slip on the stable adhesion of gecko [53]. Although the influences of factors such as peeling angle, adhesive length and pretension on the peeling force are understood based on the above models, these models also have some deficiencies to varying degrees. For example, ignoring the specific structural shape of the fiber and its corresponding material nonlinearity are their common shortcomings.

To more accurately simulate the peeling motion of gecko spatula, Sauer et al. constructed a three-dimensional simulation model based on the framework of nonlinear finite element [54]. The model takes into account the precise geometry of the spatula and the real Van der Waals interaction between the spatula and the interface. Although the model simultaneously captures the coupling effect of the geometric nonlinearity, the material nonlinearity and the contact nonlinearity, the study only simulates the peeling effect of the spatula under the action of the rotation torque and the vertical peeling force, and does not consider a more realistic situation where the normal and tangential forces are coupled during the peeling process of spatula. Meanwhile the interfacial adhesion expressed by the potential function of Lennard-Jones in the model ignores the tangential friction. Considering that the spatula involves the large deformation and large rotation in the process of attachment and detachment, the research team has also constructed a new nonlinear finite beam element model through combining the geometrically exact beam theory and two different characterization mechanism of interfacial adhesion [55]. The model takes into account the shear deformation of the beam and the bending moment caused by the adhesion which are not considered by the previous models. In view of this, based on the two different expression modes of interfacial adhesion in the nonlinear finite beam element model, the optimization models of geometric structure of the spatula in the normal peeling and the mixed peeling modes were constructed to obtain the optimal structure of spatula which meets the requirement of strong adhesion and the requirement of strong adhesion and easy separation $[56,57]$. The two optimization models are of great significance to the understanding of the role of the gecko's spatula in its adhesive characteristics, and the final optimized structures of spatula obtained by the two models are very similar to the actual shape of gecko's spatula. Nevertheless, these models still have some areas to be improved. For example, the current models only consider the linear elastic materials, so they are necessary to be extended to consider the nonlinearity of the material; the current models do not consider the coupling effect of interfacial adhesion and friction. To capture the frictional behavior during the normal adhesive process, the research team also proposed two continuous contact models that coupled the adhesion and the friction based on whether the sliding friction is dependent on the normal distance of the adhesive interfaces [58]. Based on the two models, the peeling behaviors of the strip that simulates the peeling of the spatula were explored again, and some conclusions that are similar to the gecko adhesion could be obtained: the sliding of the spatula near 
the peeling front can pre-stretch the spatula, thereby increasing the peeling force; the peeling of the spatula exists the critical detachment angle; the spatula can be easily detached from the substrate by changing the shaft angle and adopting a vertical peeling method $[59,60]$. Although the two models have been extended to three dimensions by researchers [61], the assumption that the static friction threshold based on the macroscopic measurement is consistent with the resistance for kinetic friction still needs to be verified on the local contact area. In addition, although the two models couple with the adhesion and the friction, as the researchers emphasized, the tangential friction is dependent on the normal adhesion rather than that the normal adhesion is dependent on the tangential friction in the two coupled models, which is just the opposite of the mechanism of frictional adhesion of gecko's foot. Nevertheless, these models still have important reference significance for constructing a unified model describing the mechanism of frictional adhesion of gecko.

\section{Gecko-Like Dry Adhesive Surfaces}

The adhesive function of gecko's foot is mainly derived from the Van der Waals force, and the outstanding adhesive characteristics mainly depend on the unique movement form and multi-scale layered structure of gecko's foot. Therefore, the researchers have been focusing on designing the microstructures of gecko-like dry adhesive surfaces in the research process of gecko-like dry adhesive surfaces. According to the different forms of microstructures, the microstructures on the gecko-like dry adhesive surfaces have undergone evolution from upright to inclined, from capless to capped, from uniform cross-section to variable cross-section, and from single-layer structure to multi-layer structure. The source of this evolution is mainly from the continuous pursuit for reproducing the excellent adhesive characteristics of gecko's foot. Based on the aforementioned review about the relevant adhesive characteristics of gecko's foot, the following will mainly describe and summarize the existing gecko-like dry adhesive surfaces in view of three characteristic categories: controllable adhesive characteristic, self-cleaning characteristic, and the adaptive characteristic for rough surface.

\subsection{The Gecko-Like Dry Adhesive Surfaces with the Controllable AdhesiveCharacteristic}

The controllable adhesive characteristic of gecko-like dry adhesive surfaces describes the ability to quickly switch the state of attachment and detachment and even to fine adjust the adhesion. The gecko-like dry adhesive surfaces with the controllable adhesive characteristic only need low preloading for attachment and low peeling force for detachment.
As a consequence, they basically do not disturb or affect the target surface, which is of great significance to the practical application in the fields of climbing robot and grasping the target [62].

A large number of studies have shown that the adhesive controllability of gecko's foot stems from its anisotropic adhesive characteristic on the one hand [37,63], and on the other hand, it is controlled by the unique stretching and curling movement of gecko's foot [26, 64]. However, the anisotropic adhesive characteristic of gecko's foot depends on the initial inclined configuration of its seta $[16,19]$. Based on these inspirations, designing anisotropic microstructure (such as tilted and asymmetrical microstructures) is an important means to realize the controllable adhesive characteristics of gecko-like dry adhesive surfaces. The team led by Metin Sitti is the first to combine the angled lithography based on the SU-8 photoresist and the micro-molding technique to produce the polyurethane-based cylindrical microfibers with the tilt angle of $72^{\circ}$ (as shown in Fig. 4a) [65]. Although this study does not prove the controllable adhesive characteristic of the inclined microfibers, it is the first breakthrough in the preparation of the inclined microfibers. Of course, it is still a challenging task to manufacture a uniform microfiber arrays with larger length diameter ratio and greater inclination because of the constraints of the technology (such as UV diffraction). In addition, the microfibers with high length diameter ratio are prone to breakage during demolding. In the same year, the team led by Mark Cutkosky used the machining method with multi-process to create an anisotropic microstructure called directional polymer stalk, which has the inclined feature at both the base and the end face (as shown in Fig. 4b) [62, 66]. The corresponding adhesive tests showed that the microstructure exhibits the characteristic of friction adhesion similar to that of gecko's foot, that is, the corresponding normal adhesion can be adjusted by the tangential friction. The microstructure is the first to reproduce the controllable adhesive characteristic of gecko's foot, and it has also successfully allowed the gecko-like climbing robot to climb vertically on a smooth glass surface. However, there is still room for further improvement in the scale to adapt to the rougher surface.

Although the tilted feature of microstructure is an effective means to reproduce the controllable adhesive characteristic, simply relying on the tilted feature is not enough to provide effective adhesive capacity. The actual gecko's seta also has a spatula-shaped microstructure at the end of its inclined feature, which makes an outstanding contribution to its strong adhesion. Relevant studies also confirmed that the end shape of microstructure is important in the design process of gecko-like dry adhesive surface [67], and the microstructure with spatula-shaped tip has better adhesive capacity $[43,68,69]$. To make the gecko-like dry adhesive surface have higher adhesive capacity on the basis of reproducing 


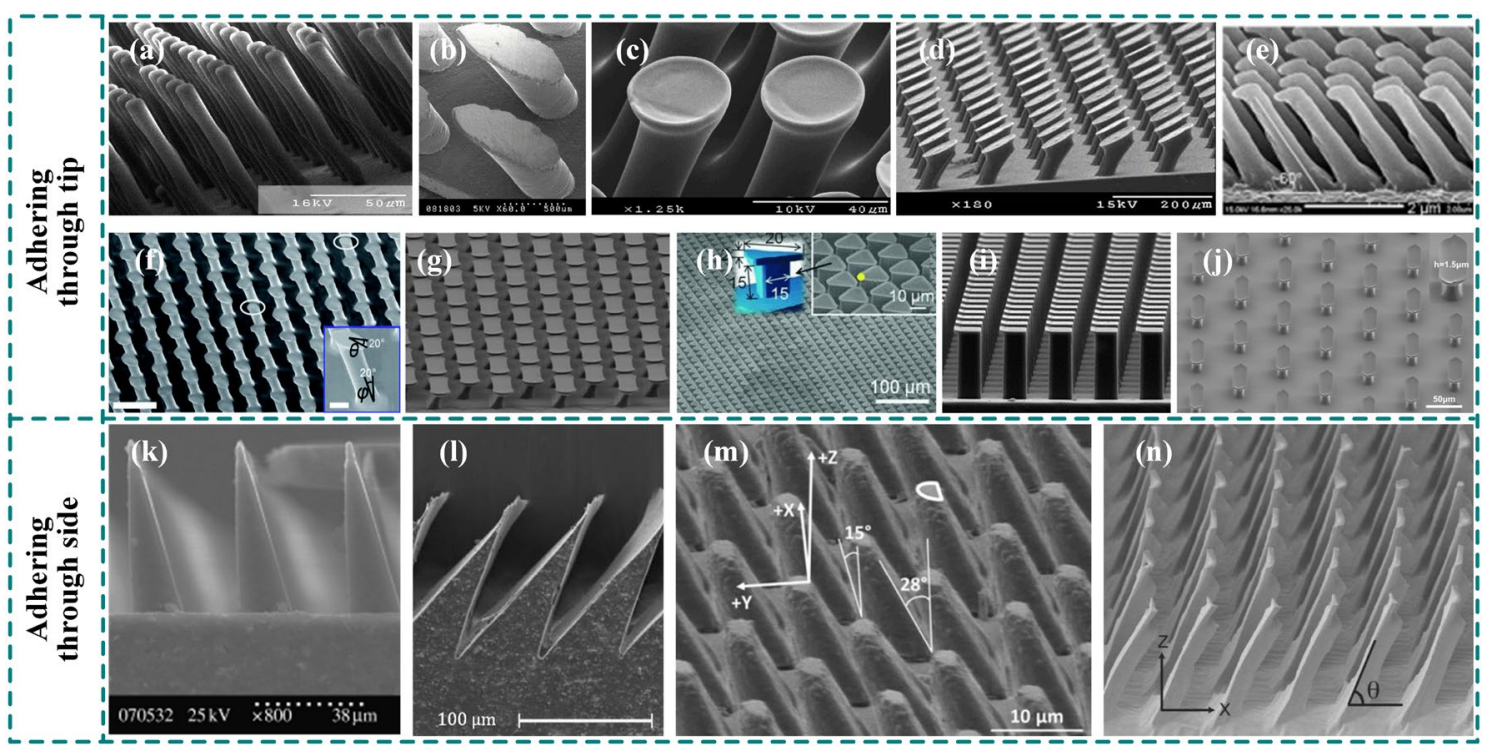

Fig. 4 The gecko-like dry adhesive surface based on the mechanism of shear induction: a cylindrical polyurethane microfibers with the tilt angle of $72^{\circ}$ [65]; $\mathbf{b}$ anisotropic microstructures called directional polymer stalk [66]; c inclined fiber with a spatula-shaped tip feature [70]; $\mathbf{d}$ inclined fiber structure with the inclined mushroomshaped tip [72]; e angled nano-hairs with a bulged flat top [64]; f double-inclined microfiber structure [73]; $\mathbf{g}$ inclined fiber structures

the controllable adhesive characteristic, a relatively simple and effective method is to carry out further tip modification or improvement on the base of simple inclined microstructure. The team led by Metin Sitti obtained a spatula-shaped tip at the top of the inclined microfiber based on an additional dipping process (as shown in Fig. 4c). The addition of the spatula-shaped tip not only increases its normal adhesion and shear friction several times, but also makes the microstructure with the controllable adhesive characteristic [70, 71]. In particular, the tangential adhesion of inclined microstructure with the inclined mushroom-shaped tip prepared in the later period (as shown in Fig. 4d) reached $100 \mathrm{kPa}$, which reached the lowest level of the tangential adhesive capacity of the gecko's foot [72]. The study once again confirms the potential of the tip modification to enhance the adhesion of microstructure, especially the tangential adhesive strength. However, the corresponding normal adhesive capacity needs to be further improved.

Carefully focusing on the scale of the aforementioned several inclined microstructures, it can be found that these geometric features are between tens to hundreds of microns, while the actual gecko's setae are at the nanometer level. Therefore, the poor adhesion of these artificial microstructures with the scale of micron may be due to their relatively large scale of structure, because studies have shown that smaller feature sizes will produce higher adhesive capacity [34]. To scale these similar with square caps [74]; $\mathbf{h}$ vertical microstructure array with equilateral triangle tip [75]; i vertical asymmetrical microstructure with a spatula-shaped tip feature [76]; $\mathbf{j}$ micro-pillar with large overhanging cap [77]; k vertical wedge-shaped microstructure [26]; $\mathbf{l}$ inclined wedgeshaped microstructure [79]; $\mathbf{m}$ inclined semi-cylindrical microfiber structure [83]; $\mathbf{n}$ double-layer inclined microstructure with tri-prism base [85]

microstructures to the nano-scale, the angled nano-hairs with a bulged flat top were fabricated through the angled plasma etching technique and replica molding, which is similar to the spatula at the end of gecko's seta in scale and morphology (as shown in Fig. 4e) [64]. The results confirmed that the nano-scale structures exhibit strong directionality in the tangential adhesion, that is, the shear adhesion in the inclined direction of nano-hairs is up to $26 \mathrm{~N} / \mathrm{cm}^{2}$, while the shear adhesion in the opposite inclined direction of nano-hairs is only $2.2 \mathrm{~N} / \mathrm{cm}^{2}$. However, the normal adhesion of angled nano-hairs was not reported. At the same time, the micro-nano-layered fibers were further fabricated using the two-step UVassisted capillary force lithography [64]. The layered fibers show certain advantages when it adapts to a rougher surface, but they show weaker tangential adhesive capacity when facing a relatively flat surface, which may be due to the low density of fiber. Similarly, the double-inclined microfibers in which both the base and the tip features are inclined were manufactured by drawing on the angled etching technique and combining the micro-molding and tip modification technology (as shown in Fig. 4f) [73]. At the same time, the double-sided adhesive arrays were further obtained by antisymmetric curing and bonding of the two single-layer adhesive arrays. Compared with the larger scale microstructures, the adhesive capacity of the microfibers with the few microns in size obtained in 
this study is indeed improved due to the smaller size. In addition, the research results also confirmed that both the monolayer adhesive arrays and the antisymmetric bilateral adhesive arrays obtained by the study exhibited obvious anisotropic adhesive characteristic and controllable adhesive characteristic, as well as their adhesive state can be controlled by switching the gripping direction. In addition, the improvement in the adhesive capacity of the antisymmetric bilateral adhesive arrays compared to the monolayer adhesive arrays also confirmed that designing the double-layer inclined microstructures further reduces the equivalent rigidity of the entire structure, which is beneficial for achieving close contact between the microstructures and the substrate. The dipping process is a relatively simple method to modify the tip feature of microfiber, but the process is not easy to quantitatively control the consistency of the lateral shape and spatial distribution of the tip due to the existence of material reflow and the nonabsolute alignment errors in the dipping interface. With this in mind, the team led by Jinyou Shao fabricated the inclined microstructures with square caps directly based on the bilateral exposure photolithography process and the molding process without using the dipping process (as shown in Fig. 4g) [74]. Since the microstructure also has the inclined axis and asymmetrical square caps on the tip, it can switch the state of the attachment and detachment through changing the shear direction.

Although designing the microstructures with inclined feature and flat cap is the mainstream in developing the gecko-like dry adhesive surfaces, there are also several special anisotropic microstructures that have been developed [75-77]. These microstructures can also exhibit anisotropic adhesive characteristic based only on the anisotropic design of the tip without the inclined base, so that they can realize the control of the adhesive state by switching the tangential loading direction. The anisotropic adhesive characteristic of vertical microstructure arrays with equilateral triangle tip (as shown in Fig. 4h) is related to the crack propagation line in the contact area of the triangle tip, and when the peeling is performed from one edge of the triangle tip to the opposite vertex, the microstructure shows stronger adhesive capacity, and vice versa [75]. However, it could be because the point contact and the line contact in the triangular contact area cannot be clearly distinguished, the anisotropic adhesive characteristic of microstructures is not significant. A vertical microstructure arrays also make its adhesive strength show anisotropic trend based only on its asymmetrical design that the spatula-shaped structure in the tip only extends to one side of the base feature (as shown in Fig. 4i), and their anisotropic adhesive characteristic is affected by the peeling angle [76]. It may be due to the fact that its base feature is upright, the microstructure arrays require a relatively large normal preload $(2 \mathrm{~N})$ to produce effective adhesion. Furthermore, the introduction of a micro-step in the centerline of the tip of a micro-pillar with large overhanging cap (as shown in Fig. 4j) can also control the actual contact area by switching the dragging direction of tangential load, so as to switch the adhesive state [77]. Compared with the completely flat symmetrical tip, the presence of step limits the maximum contact of the microstructure tip, so its corresponding adhesive capacity will be weakened.

Looking at the above-mentioned microstructures, it can be found that these microstructures are basically using the tip features for adhering. In the study of reproducing the controllable adhesive characteristic of gecko, some special microstructures have been developed, which do not possess large tip feature but use the side for adhering. Among them, the wedge-shaped microstructure is the most representative type of microstructure that use the side for adhering. Under the action of external load, especially tangential load, the wedge-shaped microstructure bends in the right direction to increase the contact area with the target interface, resulting in the gradually increased adhesion. Since this kind of wedge-shaped microstructure has at least one inclined hypotenuse, and the hypotenuse is usually the side that produces effective adhesion, it can not only exhibit anisotropic adhesive characteristic according to the switching of the tangential loading direction, but also exhibit the feature of friction adhesion similar to that of gecko adhesion. As a result, the wedge-shaped microstructure can not only easily switch the adhesive state based on the characteristic of anisotropic adhesion, but also achieve fine control of the normal adhesion through the tangential adhesion. The vertical wedgeshaped microstructure was fabricated by the dual-side and dual-angle lithography process using SU-8 photoresist (as shown in Fig. 4k) [26]. Since the wedge-shaped microstructure has a vertical side, its corresponding flexibility is not high, and its actual adhesive capacity is reduced due to the non-alignment problem of the adhesive interface under the actual uncontrollable application environment. To make up for the lack of adhesive capacity of the vertical wedgeshaped microstructures as much as possible, a double exposure angled lithography technique [78] and a micromachining process [79] were successively developed to produce the inclined wedge-shaped microstructures (as shown in Fig. 41). The inclined wedge-shaped microstructures have lower equivalent stiffness than the vertical wedge-shaped microstructures due to the appearance of the second inclined surface. Thus, the inclined wedge-shaped microstructures can better conform to the target surface. The research also confirmed that the inclined wedge-shaped microstructures fabricated by the polydimethylsiloxane have both the anisotropic and controllable adhesive characteristic, and its corresponding adhesive strength has also been significantly improved [79]. Nevertheless, both of the two processes for fabricating the inclined wedge-shaped microstructure face the problem 
that the corresponding forming molds are nondurable. To solve the problem, an addition electroplating process was developed to manufacture the metal mold based on the waxforming mold [80]. The results showed that the inclined wedge-shaped microstructures fabricated by the metal mold have no significant difference in appearance or adhesive performance from the inclined wedge-shaped microstructures fabricated by the wax-forming mold. In addition, the strategy of ultra-precision diamond fly-cutting [81] and the strategy of ultra-precision multi-step and layered scribing [82] were also successively proposed to fabricate the metal molds for directly replicate the inclined wedge-shaped microstructures. The disadvantage is that the adhesive capacity of the inclined wedge-shaped microstructures prepared by the two strategies is slightly inferior, and the former cannot produce large-scale arrays of wedge-shaped microstructures. In addition to designing the inclined wedge-shaped microstructures, the inclined semi-cylindrical microstructures (as shown in Fig. $4 \mathrm{~m}$ ) were also designed to improve the performance of the vertical wedge-shaped microstructures [83]. Apart from the above-mentioned single-layer microstructures based on the side contact, two double-layer inclined microstructures based on the side contact were also proposed [84, 85]. One of the microstructure has the cylindrical base, while the other has a tri-prism base (as shown in Fig. 4n). These inclined base features mimic the axis of gecko's seta, thereby providing compliance for the entire microstructure. In addition, at the ends of these bases, a square tip is extended to imitate the spatula of gecko, thereby serving as an adhesive unit. Compared with a single inclined cylindrical microfiber, the two square tips based on side contact not only provide local compliance for the microstructure arrays, but also maximize the area of adhesive contact.

The above-mentioned various inclined or anisotropic microstructures are based on the switching of the shear loading direction to change the morphology of microstructure, so as to realize the switch control of strong attachment and easy detachment, which is inspired by the rapid switching mechanism of gecko adhesion. In addition to these, some other activation methods were proposed to switch the adhesive state of the microstructures. The array of vertical cylindrical micro-pillars constructed by the shape memory thermoplastic elastomer can change the inclination or deformation of the micro-pillars through the temperature stimulation, so as to switch between adhesion and non-adhesion [86, 87]. The end of the multi-scale microfibers with nickel microcantilever can rotate laterally under the action of an external magnetic field, so that the adhesive state can be changed by greatly reducing the contact area [88]. The vertical columnar microfiber (with a tip cap) mixed with $\mathrm{NdFeB}$ microparticles can also produce uniform and reversible bending deformation under the action of the adjustable magnetic field, and the corresponding deformation degree is affected by the intensity of the magnetic field [89]. Therefore, its corresponding adhesion can be modulated by changing the intensity of the magnetic field. The slanted capped nanopillar made by thermal plastic material with high modulus can dynamically adjust the inclination under the action of $\mathrm{AC}$ electric field, and the maximum deflection at the end of nano-pillar exceeds ten microns, which is also promising for the hard fiber to achieve reversible adhesion [90]. In addition, the vertical cylindrical microfiber fabricated by the shape memory polymer mixed with graphene can also adjust its storage modulus by the switch of the UV-irradiation or the cycle of cooling and heating, so as to indirectly switch the adhesive state [91]. Studies showed that changing the stiffness of the backing layer of the dry adhesive surface can also affect its adhesive strength: the low stiffness of the backing layer at the initial time of contact can promote the fibers to better adapt to the surface, thereby forming a close contact; after the contact is completed, the transition of the backing layer to high stiffness can impel the load distribution between the fibers to be more uniform, resulting in high adhesive strength $[92,93]$. On these grounds, some researchers realized the regulation of adhesive state by inducing the change in the stiffness of the backing layer of dry adhesive surface. By embedding different phase change materials in the backing layer of mushroom-shaped microfibers, the stiffness of the backing layer can be adjusted by the transformation of the phase change materials in the two phase change states $[94,95]$. By mixing iron oxide particles in the backing layer of mushroom-shaped microfibers, the stiffness of the backing layer can also be adjusted by the application of the magnetic field [96].

The controllable adhesive characteristic of the gecko-like dry adhesive surfaces is necessary for its practical applications. These active activation strategies such as temperature field, magnetic field, and electric field provide new ideas for the switch control of the adhesive state of gecko-like dry adhesive surfaces. However, compared with the shearinduced mechanism, they are not easy to achieve refined control on the adhesion of gecko-like dry adhesive surfaces, and they also require additional energy source. In addition, the shear-induced mechanism generally also possesses a higher control efficiency, so it may still be the main mechanism to make the gecko-like dry adhesive surfaces have the controllable adhesive characteristic for a period of time in the future. To better compare the difference in adhesive capacity between gecko-like dry adhesive surfaces that achieve the controlled adhesion based on the shear-induced mechanism, they are summarized deliberately in Table 2 . It can be clearly seen from the table that the inclined microstructures with square caps and the inclined wedge-shaped microstructures have the most prominent normal adhesive capacity and tangential friction capacity, and the coefficient of adhesion of the former has reached the lowest level of the coefficient of 


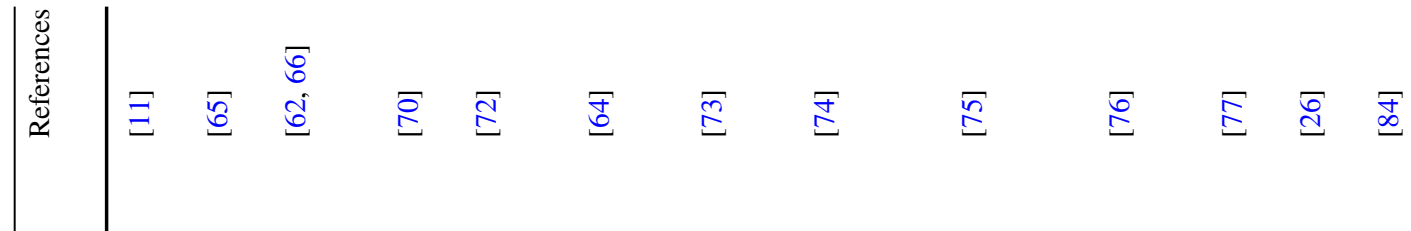

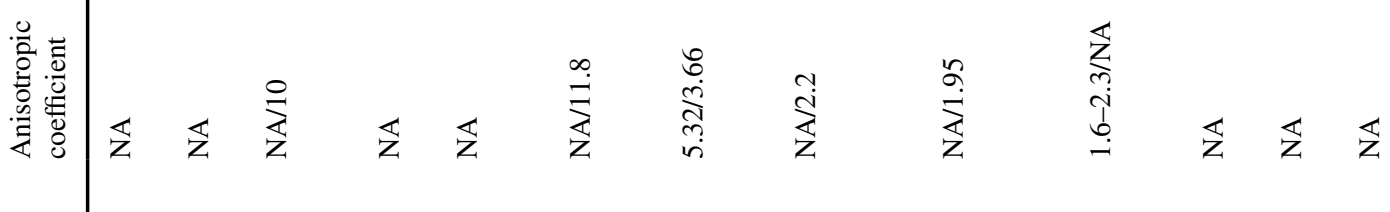

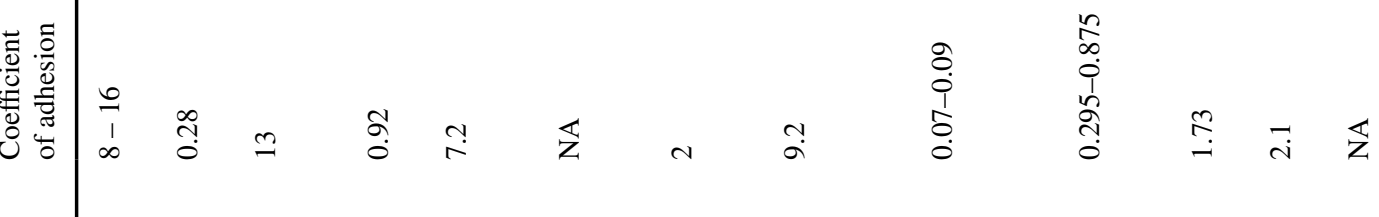

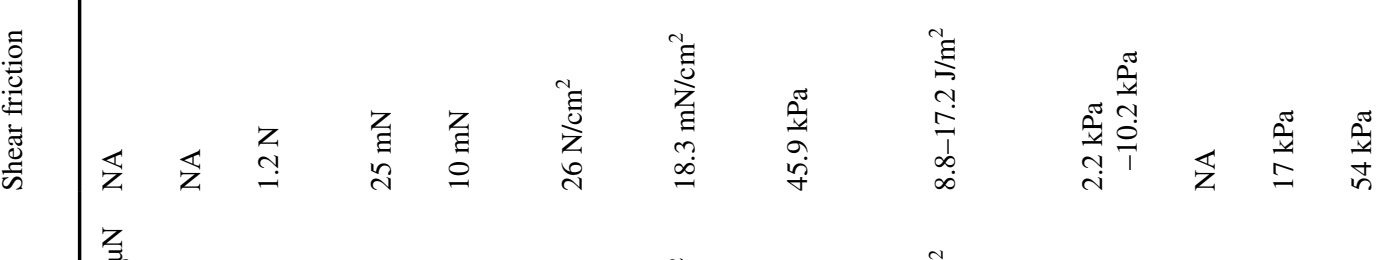

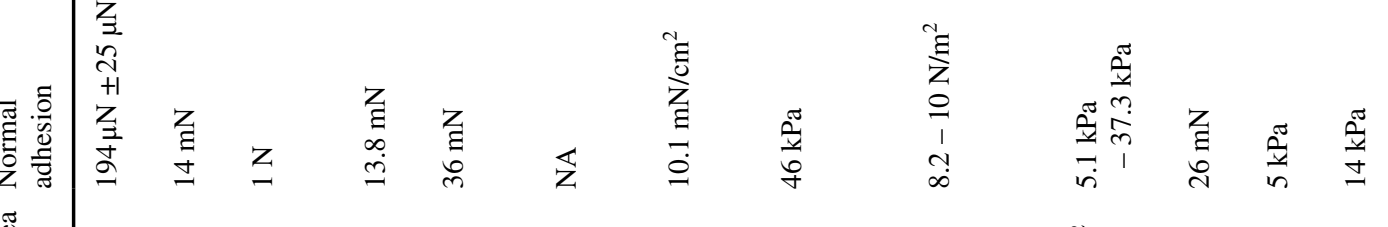

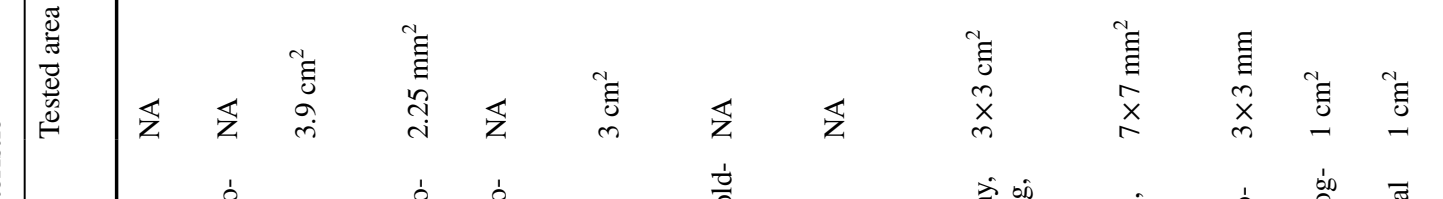

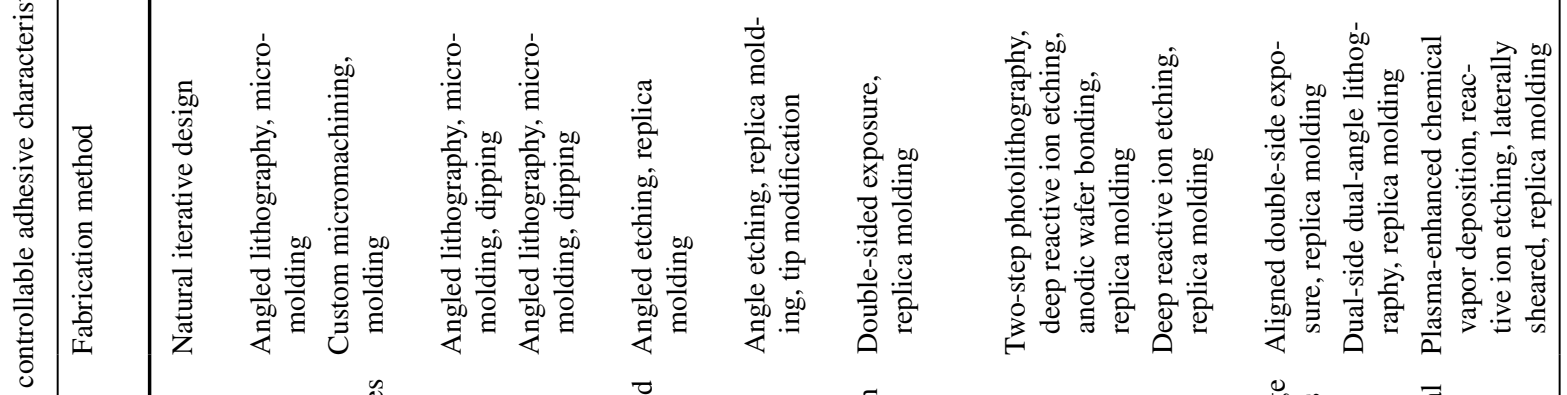

$$
\begin{aligned}
& \text { 馬 }
\end{aligned}
$$

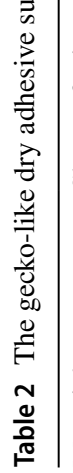

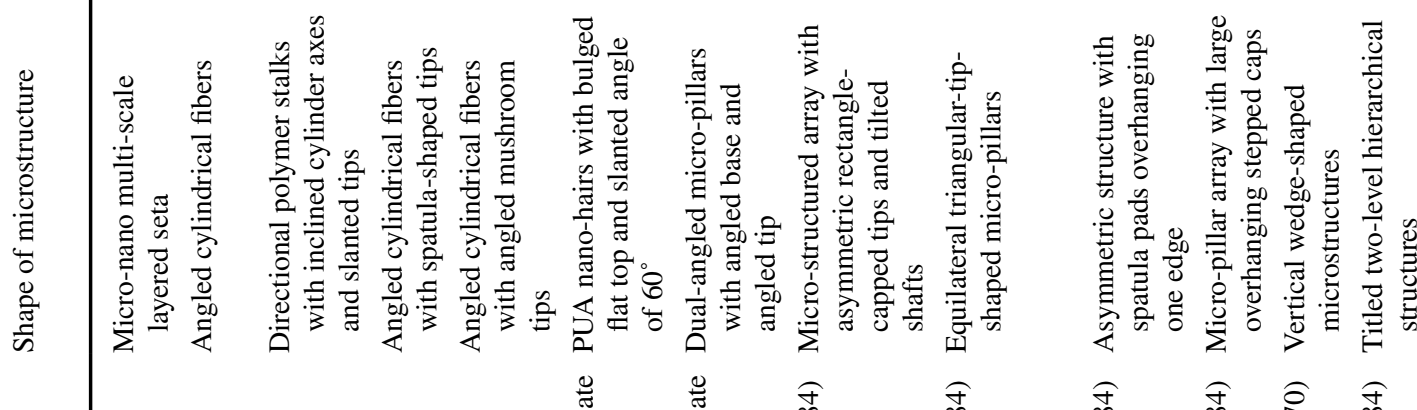

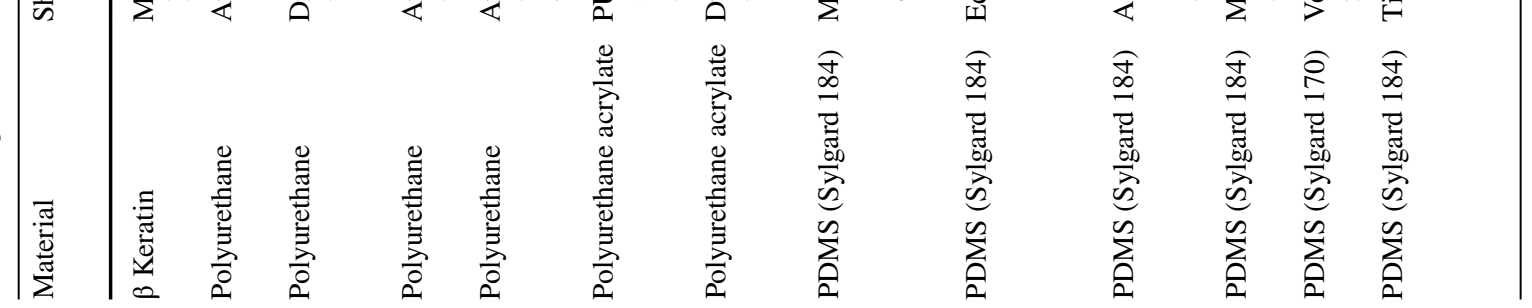$$
\text { 莺 }
$$ 
adhesion of the gecko foot. Although the coefficient of adhesion of the inclined wedge-shaped microstructures has not been reported, it has been confirmed in subsequent application studies that it only requires a low preload for adhesion. In view of these, further improvements can be carried out based on the basic features of the two microstructure arrays (inclined and having the cap, inclined and variable crosssection, etc.) to create a gecko-like controllable dry adhesive surface with more outstanding adhesive performance in the future.

\subsection{The Gecko-Like Dry Adhesive Surfaces with the Self-Cleaning Characteristic}

The gecko-like dry adhesive surfaces will inevitably be contaminated due to the presence of dirty particles in the process of actual use, which will have a serious impact on its adhesive capacity. Therefore, making the gecko-like dry adhesive surfaces with self-cleaning characteristic is of great significance for ensuring its long-term and effective adhesive capacity in the real environment. Drawing lessons from the three methods behind the self-cleaning of gecko's foot, namely wet self-cleaning, contact self-cleaning and dynamic self-cleaning, the self-cleaning abilities of some gecko-like dry adhesive surfaces have been studied.

The wet self-cleaning is the easiest self-cleaning way to be directly used for the gecko-like dry adhesive surfaces, because its essence is based on the hydrophobic and even super-hydrophobic characteristic of the dry adhesive surfaces, which is similar or equivalent to the lotus effect (as shown in Fig. 5a). It is precisely because most of the gecko-like dry adhesive surfaces are hydrophobic or even super-hydrophobic because of its unique micro-nano-scale structure, so most of gecko-like dry adhesive surfaces should be able to achieve wet self-cleaning. At present, the wet self-cleaning method has been successively verified on the gecko-like dry adhesive surfaces fabricated by the carbon nanotubes [97], polyurethane [98], polyethylene [99] and polydimethylsiloxane [100], and their corresponding selfcleaning effect is in the range of $60 \%-100 \%$ (see Table 3 for details). At the same time, these studies also indicated that the hydrophobic or super-hydrophobic properties of the gecko-like dry adhesive surface could be further optimized by modifying the micro/nano-scale structure on the surface (such as modifying the tip geometry [98] and modifying the size and the duty cycle [100]). Since the wet self-cleaning method requires the participation of water droplets, the method is not suitable for environments where water is not available (such as the outer space).

The contact self-cleaning is based on the contact between the gecko's foot and the interface to remove the dirty particles. Simulating the contact process of gecko's foot, the contact self-cleaning method was verified on the gecko-like 

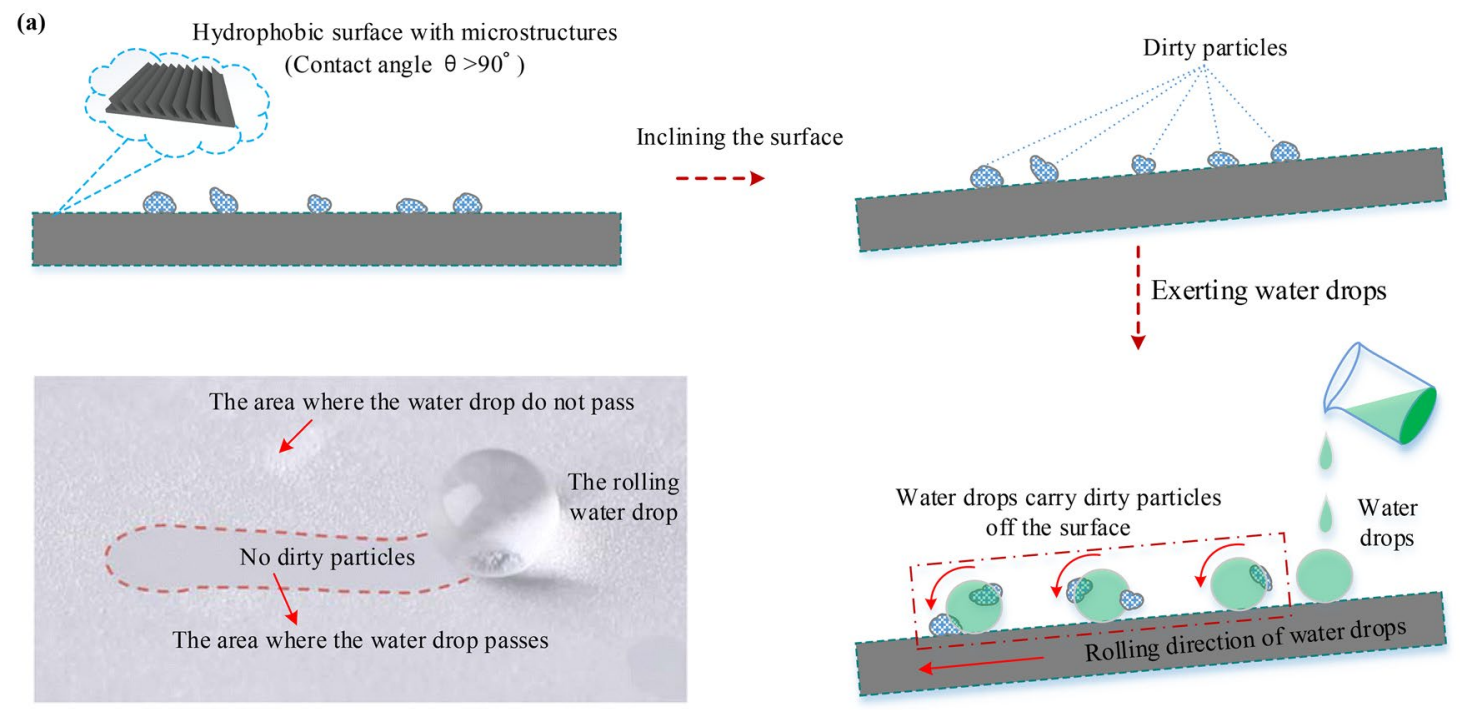

(b)
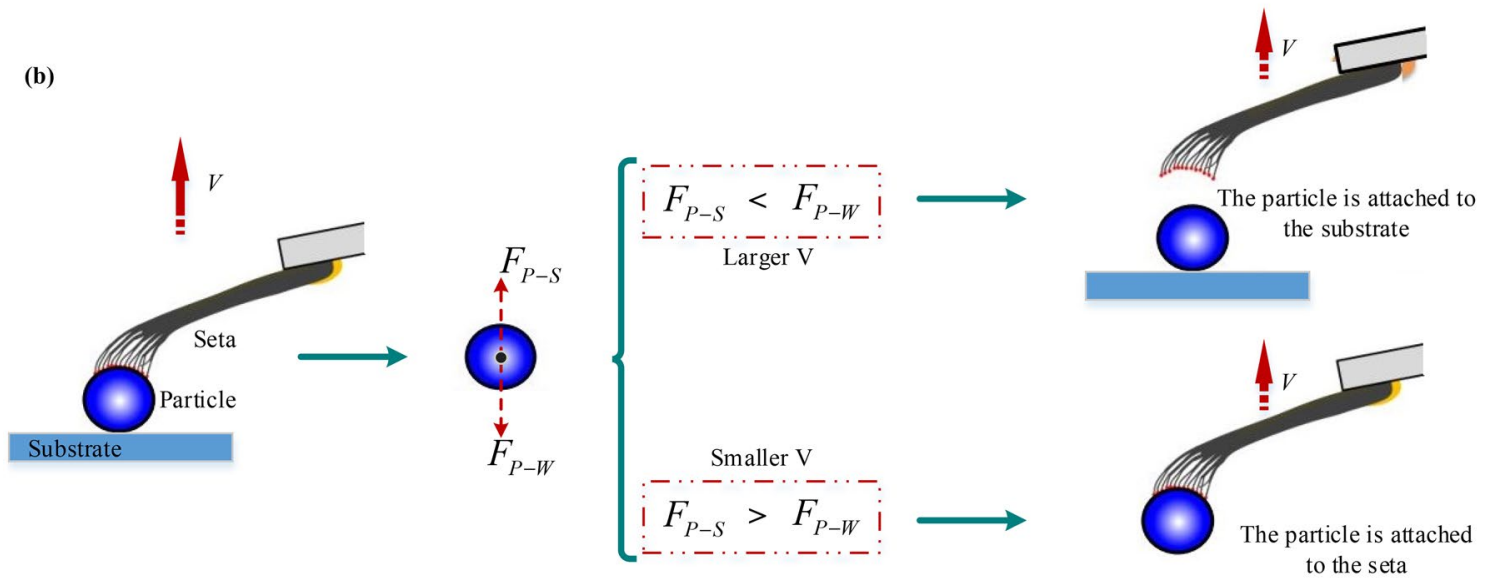

Fig. 5 The schematic diagram of wet self-cleaning and dynamic self-cleaning. a The particles roll away from the super-hydrophobic surface with the roll of droplets in the process of wet self-cleaning [99]. b The dynamic self-cleaning gets benefit from the difference in response to the normal peeling speed $(V)$ between the adhesion of the substrate to the particles $\left(F_{P-W}\right)$ and the adhesion of the seta to the particles $\left(F_{P-S}\right)$ : when the normal peeling speed is large enough

dry adhesive surfaces (see Table 3 for details), and some factors related to the effect of the contact self-cleaning were also explored. The polypropylene-based rigid vertical microfibers with high aspect ratio have been proven to be able to achieve cleaning effect of $60 \%$ for the gold microspheres with a radius of $1.15 \mu \mathrm{m}$ through 30 contact steps. However, the research also found that the self-cleaning ability of the microfibers is dependent on the size of the messy particles, and the microfibers cannot clean the polystyrenebased microspheres with radius greater than $2 \mu \mathrm{m}$ [101]. The different contact self-cleaning effect of hard-polymer microfibers on messy particles with different sizes can be explained by the imbalanced adhesion between microfibers, to make the adhesion of the substrate to the particles larger than the adhesion of the seta to the particles, the particle are finally attached to the substrate after the seta is peeled vertically; when the normal peeling speed is not enough to make the adhesion of the substrate to the particles larger than the adhesion of the seta to the particles, the particle are finally attached to the seta after the seta is peeled vertically [106]

particles and substrate (as shown in Fig. 6a). The particles with smaller size can only have a small amount of contact with the microfibers so that the adhesion between the particles and the substrate is dominant, so the particles will stay on the substrate after the contact self-cleaning is completed. Yet, the particles with larger size can have relatively large contact with the microfibers so that the adhesion between the particles and the microfibers is dominant, so the particles will retain on the microfibers after the contact selfcleaning is completed. Besides, the embedding of largesize particles between the microfibers will also promote the situation. Furthermore, there was also study to compare the cleaning ability of hard polypropylene nanofibers and soft 
Table 3 The gecko-like dry adhesive surfaces with self-cleaning characteristic

\begin{tabular}{|c|c|c|c|c|}
\hline Self-cleaning mechanism & Microstructures & The size of pollution particles & Self-cleaning effect & References \\
\hline \multirow[t]{4}{*}{ Wet self-cleaning } & $\begin{array}{l}\text { Vertically aligned } \\
\text { carbon nanotubes }\end{array}$ & Silica particles $(1 \mu \mathrm{m}-100 \mu \mathrm{m}$ diameter $)$ & $60 \%$ & [97] \\
\hline & Mushroom shaped & Silica particles $(5 \mu \mathrm{m}-50 \mu \mathrm{m}$ diameter $)$ & Almost $100 \%$ & [98] \\
\hline & Upright cylindrical & $\begin{array}{l}\text { Ceramic microspheres (mixed with } 1 \mu \mathrm{m}, 4 \mu \mathrm{m} \text {, } \\
15 \mu \mathrm{m} \text {, and } 24 \mu \mathrm{m} \text { diameter) }\end{array}$ & About $98 \%$ & [99] \\
\hline & Upright cylindrical & Silica particles (average diameter of $75 \mu \mathrm{m}$ ) & $91.3 \%$ & {$[100]$} \\
\hline \multirow[t]{4}{*}{ Contact self-cleaning } & Upright cylindrical & Gold microspheres (1.15 $\mu \mathrm{m}$ radius $)$ & About $33 \%$ & {$[101]$} \\
\hline & Vertical wedge-shape & $\begin{array}{l}\text { Small polystyrene microspheres }(1 \mu \mathrm{m} \text { diameter }) \\
\text { Medium glass microspheres }(3 \mu \mathrm{m}-10 \mu \mathrm{m} \text { diam- } \\
\text { eter) } \\
\text { Large glass microspheres }(40 \mu \mathrm{m}-50 \mu \mathrm{m} \text { diameter })\end{array}$ & $\begin{array}{l}99 \% \pm 4 \% \text { (small) } \\
40 \% \pm 15 \% \text { (medium) } \\
55 \% \pm 18 \% \text { (large) }\end{array}$ & {$[102]$} \\
\hline & Mushroom-shaped & $\begin{array}{l}\text { Glass microspheres with diameters ranging from } \\
3 \mu \mathrm{m} \text { to } 215 \mu \mathrm{m}\end{array}$ & $\begin{array}{l}40 \% \text { (small) } \\
55 \% \text { (medium) } \\
80 \% \text { (large) }\end{array}$ & [103] \\
\hline & Vertical wedge-shape & $\begin{array}{l}\text { Glass beads with diameters ranging from } 4 \mu \mathrm{m} \text { to } \\
212 \mu \mathrm{m}\end{array}$ & $\begin{array}{l}15 \%-53 \% \\
(4 \mu \mathrm{m}-15 \mu \mathrm{m}) \\
0(38 \mu \mathrm{m}-53 \mu \mathrm{m}) \\
16 \%-64 \% \\
(90 \mu \mathrm{m}-106 \mu \mathrm{m}) \\
15 \%-75 \% \\
(180 \mu \mathrm{m}-212 \mu \mathrm{m})\end{array}$ & {$[105]$} \\
\hline Dynamic self-cleaning & $\begin{array}{l}\text { Polyester microfib- } \\
\text { ers and graphene } \\
\text { multilayers }\end{array}$ & $\mathrm{SiO}_{2}$ micro-particles (size $\left.1 \mu \mathrm{m}-25 \mu \mathrm{m}\right)$ & $80 \%$ & {$[30]$} \\
\hline Electrostatic self-cleaning & Vertical wedge-shape & Real-world dusty & Average $76 \%$ & {$[109]$} \\
\hline Ultrasonic self-cleaning & Vertical wedge-shape & Real-world dusty & Average $79 \%$ & [109] \\
\hline $\begin{array}{l}\text { Self-cleaning combined } \\
\text { static electricity and } \\
\text { ultrasonic }\end{array}$ & Vertical wedge-shape & Real-world dusty & Average $88 \%$ & [109] \\
\hline
\end{tabular}

polydimethylsiloxane microfibers on particles with different radii, it was found that the contact self-cleaning ability of the former is more reliable than the latter. As a result, it was recommended to choose a matrix material with a low loss function and design smaller diameter of fiber to obtain a better effect of contact self-cleaning [102]. Following the similar cleaning process of load-drag-unload (as shown in the upper part of Fig. 6b), the contact self-cleaning performance of polyurethane-based mushroom-shaped microfiber arrays to particles with different diameters was also studied, and the function of the normal loading and the shear loading on the contact self-cleaning process was especially discussed. The results showed that the contact self-cleaning effect of polyurethane-based mushroom-shaped microfiber arrays strongly depends on the relative size between microfibers and particles: when the tip of microfiber is much smaller than the pollution particles, the cleaning effect is best [103]. Perhaps it is because of the dependence of relative size, particles and microfibers can appear in different geometric configurations (as shown in the lower part of Fig. 6b), so that the mushroom-shaped microfiber arrays can also achieve temporary self-cleaning by particle embedding in addition to achieving the contact self-cleaning by particles deposition like the microfibers with high aspect ratio. In addition, the results also showed that the tangential dragging behavior that simulates the contact motion of the gecko's foot is important for the contact self-cleaning under smaller or larger contaminants regime, and the main mechanism behind it is the sliding or rolling of particles on the contact interfaces, which can be preliminarily confirmed by the established approximate mechanical model (as shown in the lower part of Fig. 6c) [103]. In particular, the contact self-cleaning map based on the mechanical model showed that the sliding or rolling behavior of particles in the process of contact selfcleaning is regulated by the normal load: the larger normal load will make the rolling of particles dominate, while the smaller normal load will contribute to the sliding of particles. To further verify this point, the micro-scale behavior of particles in the process of contact self-cleaning was studied. According to the normalized particles displacement $(\rho)$, the micro-scale behavior of particles can be divided into three critical cases: sliding only on substrate $(\rho=0)$, sliding only on microfiber $(\rho=2)$ and rolling on both surfaces $(\rho=1)$ (as shown in Fig. 6d). The actual measurement results showed 

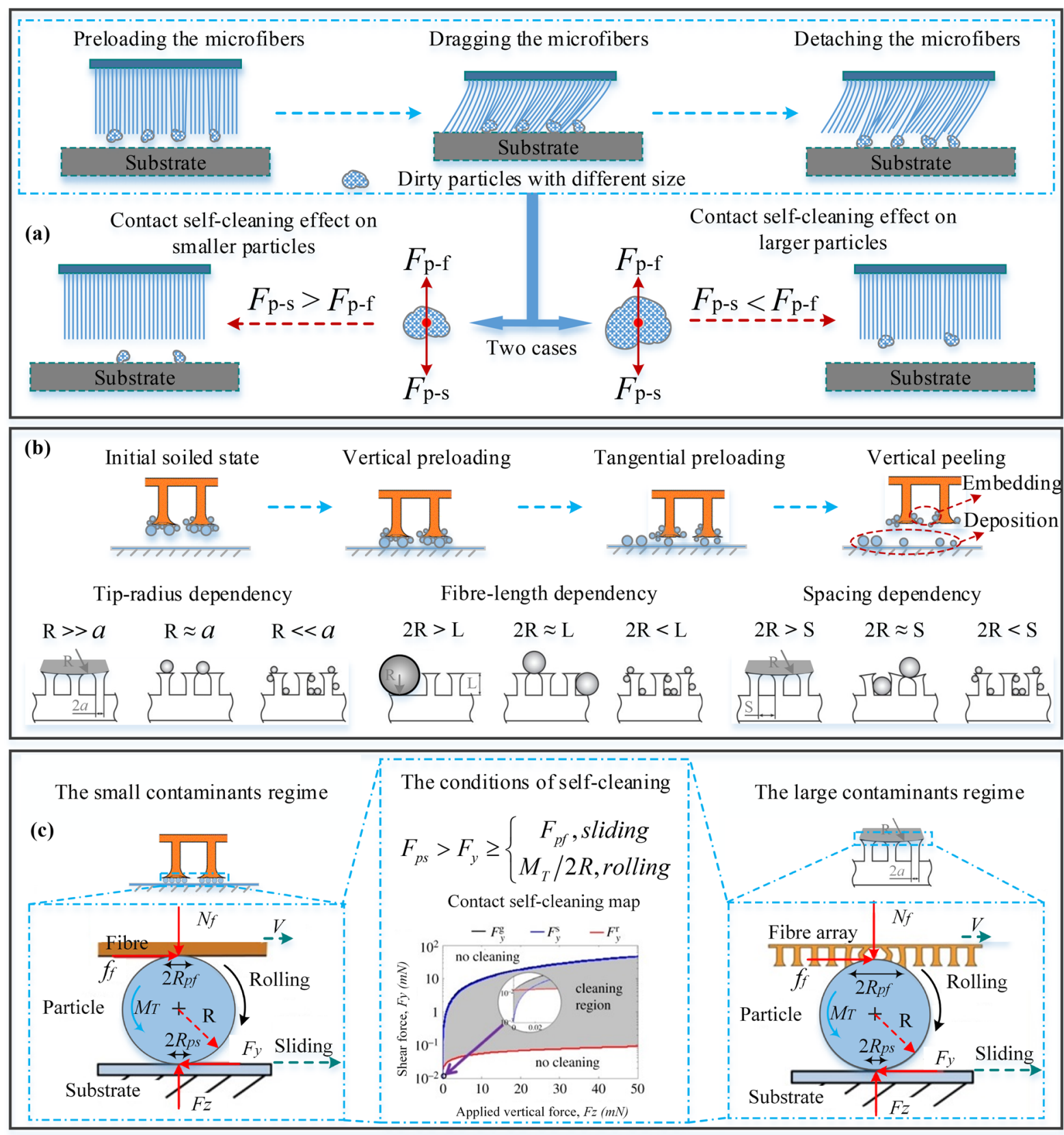

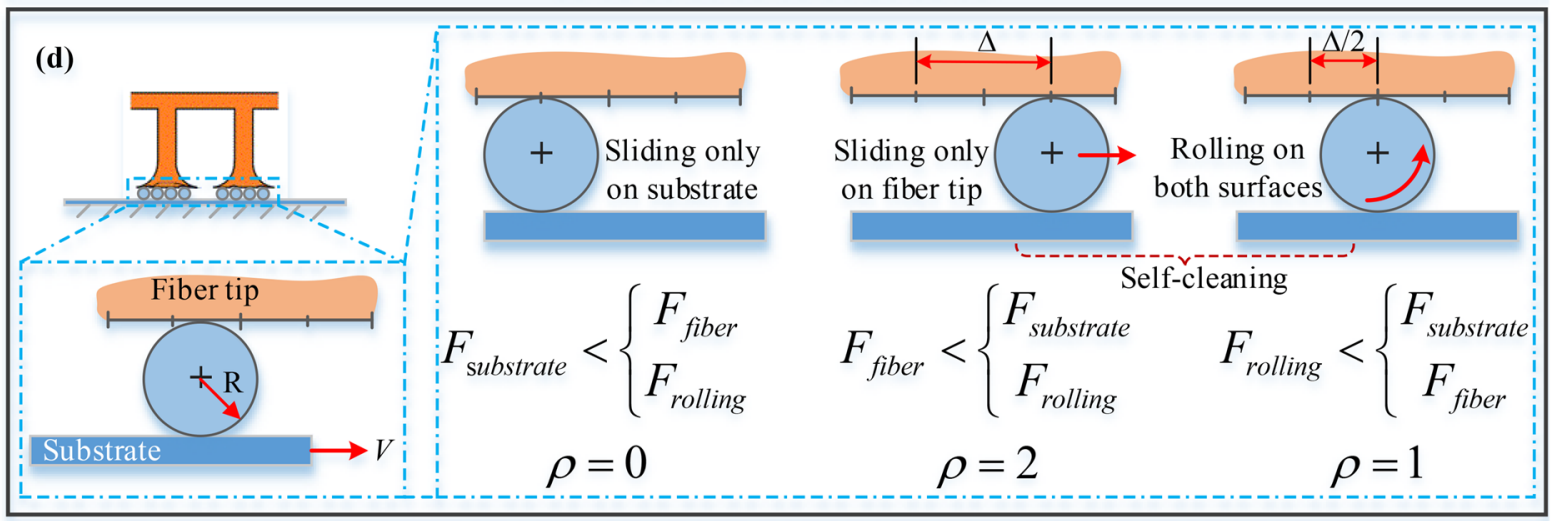


4Fig. 6 The schematic diagram of contact self-cleaning. a The contact self-cleaning process of polypropylene-based rigid vertical microfiber array with high aspect ratio and the contact self-cleaning effect on smaller or larger particles, $F_{\mathrm{p}-\mathrm{f}}$ is the adhesion between dirty particle and microfibers, $F_{\mathrm{p}-\mathrm{s}}$ is the adhesion between dirty particle and substrate [101]; $\mathbf{b}$ the contact self-cleaning process of polyurethanebased mushroom-shaped microfiber arrays to particles with different diameters and the relative geometric configurations between particles with different diameter and fibers with different geometric size [102]; c the approximate mechanical model describing contact self-cleaning of particles with smaller and larger diameter under tangential load and the corresponding contact self-cleaning map [103]; $\mathbf{d}$ the critical micro-scale behavior of particles during the process of contact selfcleaning under the tangential dragging based on the normalized particles displacement $\rho, F_{\text {substrate, }} F_{\text {fiber }}$ and $F_{\text {rolling }}$ are the respective friction force for the various motions [104]

that the normalized particles displacement $(\rho)$ always floats around 1 under the advisable normal load, which indicates the rolling of particles is more important than the sliding of particles during the process of contact self-cleaning under smaller or larger contaminants regime [104]. Besides, the study also showed that the rolling friction is affected by the applied normal load and tangential dragging speed, and the large normal load and tangential dragging speed will lead to the increase of rolling resistance [104]. Therefore, the low normal load and tangential dragging speed should be adopted to improve the effect of contact self-cleaning. Subsequently, the study also found that the matrix material with higher elastic modulus and lower adhesive work can make the vertical wedge-shaped microarray have a better effect of contact self-cleaning, and the main mechanism of the microarray to achieve contact self-cleaning is also the rolling of particles [105]. Compared with the wet self-cleaning, the contact self-cleaning may be more suitable for general environment because it does not require the intervention of water droplets. However, it often requires repeated contact on a clean substrate to have a better cleaning effect, which usually leads to the destruction of fiber structure due to the contact fatigue. In addition, the method is also affected by the relative size between the contaminated particles and the microfibers. Therefore, the contact self-cleaning will be limited in practical application.

Compared with the contact self-cleaning, the dynamic self-cleaning based on the hyperextension movement of the end of gecko's foot not only has twice cleaning efficiency, but also has a higher cleaning effect. Therefore, the dynamic self-cleaning has great potential for using on the gecko-like dry adhesive surfaces. Nevertheless, only the artificial seta composed of polyester fiber and the graphene layer can achieve the removal of micro-scale particles through the adjustment of the normal peeling speed at present (as shown in Fig. 5b) [30, 106]. Therefore, the application of dynamic self-cleaning mechanism on the gecko-like dry adhesive surfaces still needs to be further explored.

In addition to the above-mentioned self-cleaning methods inspired by the gecko, the researchers also introduced two non-destructive and non-contact cleaning methods (electrostatic self-cleaning and ultrasonic self-cleaning) to clean the gecko-like dry adhesive surfaces [107-110]. Among them, the electrostatic self-cleaning is to achieve the removal of dirty particles through the electrostatic force generated by coplanar comb-shaped electrodes (as shown in Fig. 7a), and the cleaning function benefits from the change in the signs of the Coulomb force and the dielectrophoretic force experienced by the charged particle under the action of alternating electric field (as shown in Fig. 7b) [107]. Yet, the ultrasonic self-cleaning is to complete the removal of dirty particles through the vibrations excited by the piezoelectric elements [108]. When using the method of electrostatic self-cleaning to clean the gecko-like dry adhesive surface with vertical wedgeshaped microstructures, the results showed that the electrostatic self-cleaning has the best cleaning effect on particles with a diameter in the range of $9 \mu \mathrm{m}-106 \mu \mathrm{m}$, but it is not easy to remove these particles with a diameter of less than $38 \mu \mathrm{m}$ [110]. This situation may be due to the fact that small particles are easily embedded between the wedgeshaped microstructures, and the adhesion on smaller particles is greater than the electrostatic force on them. At the same time, the results also showed that the electrostatic self-cleaning exhibits different removal effects of particle on the electrode and the electrode gap, which is mainly due to the different removal mechanism of particle on the electrode and the space between the electrodes [110]. Based on the comprehensive analysis of the force states of particle under the action of three electrostatic particle removal mechanisms (as shown in Fig. 7c) and the distribution of different electrostatic forces on the electrode and the electrode gap, it is reasonable to believe that the former is mainly achieved by the particle repulsion, while the latter is mainly achieved by the particle sliding and rolling. In addition, in view of the fact that the cleaning effect on the electrode gap is better than the cleaning effect on the electrode, it is inferred that the particle sliding and rolling are more important than the particle repulsion in the method of electrostatic self-cleaning. Ultrasonic vibration, as a very effective cleaning method, has been also proved to be able to clean the gecko-like dry adhesive surface with vertical wedge-shaped microstructures, and the corresponding vibration force to remove the particles depended on the adhesive strength between the microstructure and the substrate [108]. Moreover, the comparative study found that the cleaning effect of the ultrasonic self-cleaning is better than the electrostatic self-cleaning, especially for the smaller-scale particles [109]. Yet, the 


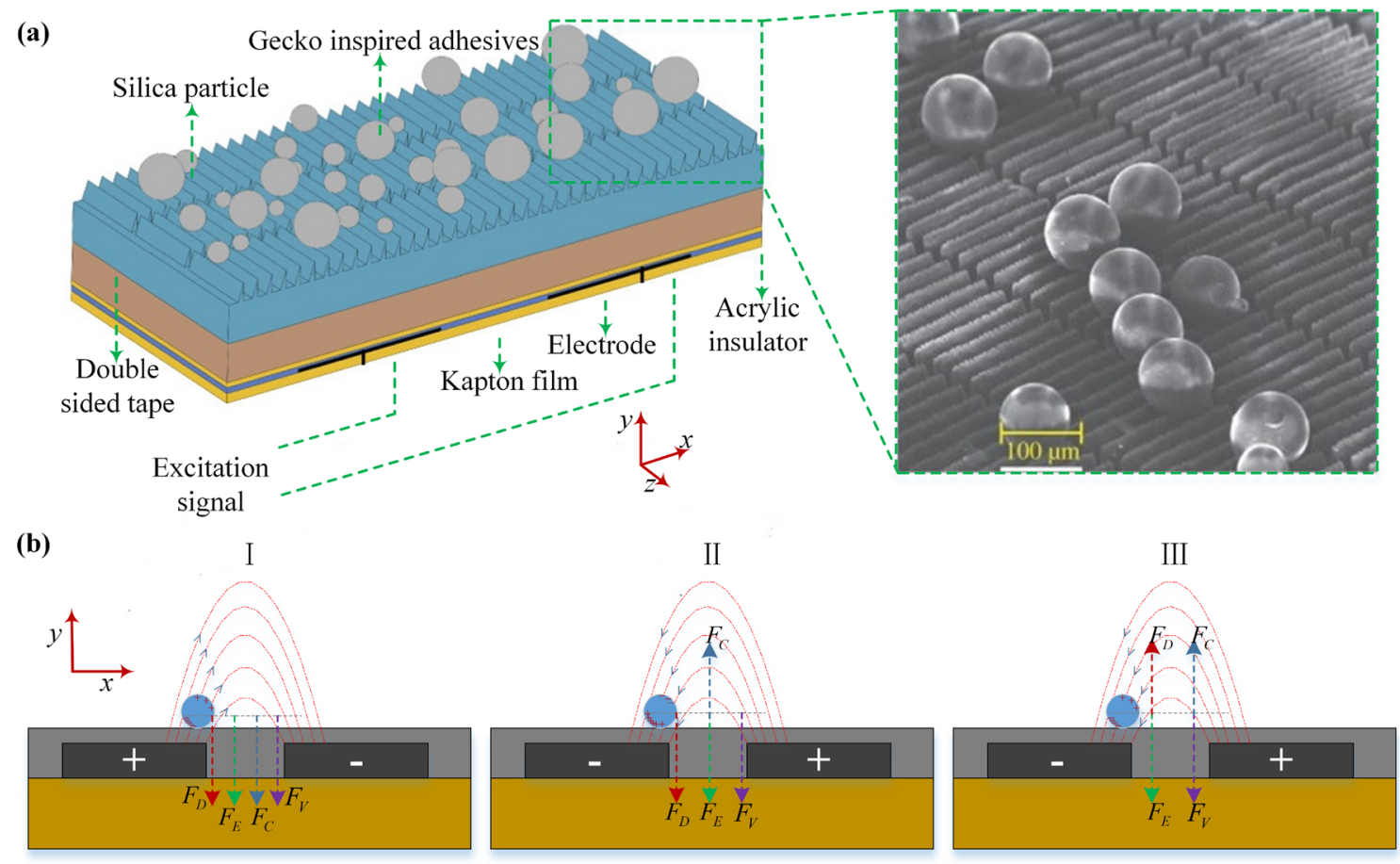

(c)

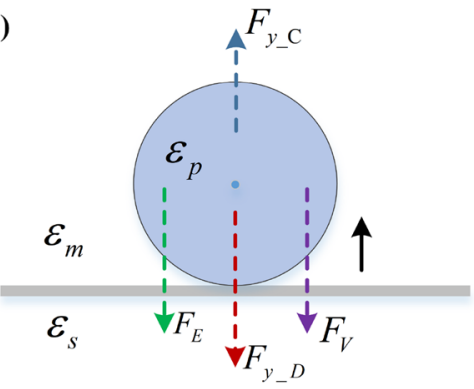

Particle repulsion

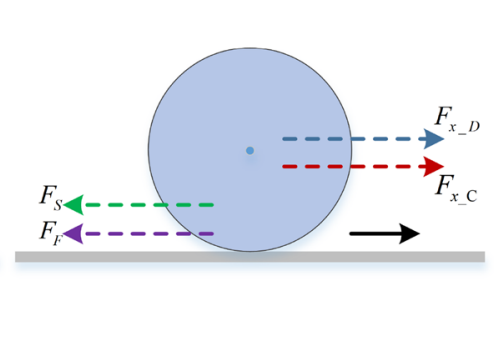

Particle sliding

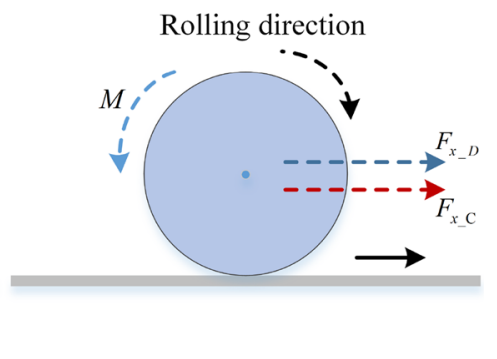

Particle rolling
Fig. 7 The configuration of gecko-like dry adhesive surface with electrostatic self-cleaning and the schematic diagram of electrostatic self-cleaning $[107,110]$. a The configuration of gecko-like dry adhesive surface with electrostatic self-cleaning. b Three force states in the Y-direction of negatively charged particle that has completed surface polarization under alternating excitation signal:I. it is assumed that the Coulomb force $\left(F_{C}\right)$ and the dielectrophoretic force $\left(F_{D}\right)$ of the negatively charged particle in the initial state are both the adhesive force, while the electrostatic image force $\left(F_{E}\right)$ and the Van der Waals force $\left(F_{V}\right)$ will always be the adhesive force because they are independent of the polarity of the electrode;II. if the polarity switching time of the electrode is longer than the dielectric relaxation time of the particle, the direction of the electric field changes and the polarization charge on the particle surface also changes, so that the

ultrasonic self-cleaning cannot be effective on the particles located on the edge of the piezoelectric element because of the edge effect of the piezoelectric element. Fortunately, it was confirmed that the cleaning effects of the electrostatic self-cleaning and the ultrasonic self-cleaning are
Coulomb force becomes the repulsive force, but the dielectrophoretic force is still the adhesive force;III. if the polarity switching time of the electrode is shorter than the dielectric relaxation time of the particle, even though the direction of the electric field changes, the polarization charge on the particle surface does not change, so that the Coulomb force becomes the repulsive force, and the dielectrophoretic force also becomes the repulsive force. $\mathbf{c}$ The force states of charged particle under three electrostatic particle detachment mechanisms, $F_{x-C}$ and $F_{y-C}$ represent the components of the Coulomb force in the $x$ and $y$ directions, respectively; $F_{x-D}$ and $F_{y-D}$ represent the components of the dielectrophoretic force in the $x$ and $y$ directions, respectively; $F_{S}, F_{F}$ and $M$ represent the shear strength, friction force and rolling resistance of particle, respectively; the black solid arrows indicate the removal direction of particle

complementary: the electrostatic self-cleaning can assist in cleaning the "particle ring" left by the ultrasonic selfcleaning, while the ultrasonic self-cleaning can improve the cleaning effect of the electrostatic self-cleaning, and their combination can significantly improve the cleaning 
efficiency and the cleaning effect (see Table 3 for details) [109]. Of course, the two self-cleaning methods can also be combined with the aforementioned wet and contact self-cleaning to further enhance the cleaning effect.

\subsection{The Gecko-Like Dry Adhesive Surfaces with the Adaptive Characteristic for Rough Surface}

Making the gecko-like dry adhesive surfaces have the ability to adapt to the rough surface is one of the ultimate goals of studying the gecko-like dry adhesive surfaces, which is also a problem that must be solved to make the gecko-like dry adhesive surfaces more widely applied. The gecko's ability to adapt to different surface morphologies benefits from the micro-nano multi-scale layered setae of its foot. The micro-nano multi-scale layered setae can give the foot of gecko compliance, so that the foot of gecko can better adapt to the rough contours of the substrate, thereby forming more contact with the rough substrate [111-113]. The multi-level layered morphology of setae on the gecko's foot is indispensable for its foot to effective adhere on the rough surface [42, 114].

Based on the inspiration of the layered adhesive system of gecko, making the gecko-like dry adhesive surfaces possess multi-level layered microstructures is one of the most direct and effective strategies to improve its ability to adapt to the rough surface. Initially, the researchers used the inductively coupled oxygen plasma to induce the growth of the organic looking polymeric nanorods on a silicon dioxide platform supported by a single single-crystal silicon pillar, thereby forming a multi-scale compliant layered structure which has been proven to promote the adhesive capacity [115]. Subsequently, some double-layer vertical cylindrical microstructure arrays were fabricated through different molding processes [116-119]. Although these double-layer vertical cylindrical microstructure arrays have a certain effect on improving the super hydrophobicity, their promotion of adhesive capacity is not obvious. In addition, the doublelayer vertical cylindrical microstructure arrays constructed from the polymer materials with low elastic modulus are generally not suitable for having a high slenderness ratio because of their collapse or aggregation. To make up for the deficiencies of the soft polymer-based materials in the preparation of the layered microstructures with high slenderness ratio, a micro-nano two-scale layered microstructure integrating the vertically arranged carbon nanotube array and the vertical cylindrical microstructure was built [120]. The carbon nanotubes generally have a higher modulus, so they can still have a high slenderness ratio without collapse or aggregation, thereby exhibiting the compliance. The results confirmed that the double-layer microstructure arrays with carbon nanotubes not only show an adhesive improvement of more than three times on the smooth surface, but also show an adhesive improvement of more than an order of magnitude on the submicron rough surface [120]. The adhesive improvements benefit from the compensation of the actual contact area by the flexibility of the layered microstructure arrays, and the compensation mechanism is consistent with the compensation mechanism of the layered structure of gecko's foot for the adhesion.

To make the artificial layered microstructures more similar to the gecko's setae, the configuration of inclined microstructure was introduced to the layered microstructures through a new process. Combining the heating rollers and the laminating process, nano-scale fibers with high slenderness ratio were replicated on millimeter-scale lamella arrays to form a layered fiber [121]. Among them, the hard-polymer lamella arrays with a certain degree of curvature mainly simulate the layered structure of the gecko's foot, which provides great flexibility for the nanofibers and makes them easier to adapt to the non-planar surfaces. The corresponding test results have also proved this point: the flexibility of the double-layer fibers with the lamella-layer array is 160 times that of the single-layer fibers without the lamella-layer array, and the difference of their adhesive strength on non-planar surfaces is five times [121]. Using the two-step UV-assisted molding technique, the micro-nano layered fibers with a protruding flat tip were prepared [64]. The layered fibers have relatively good adhesive performance on the rough surfaces, while their adhesive performance is poor on the relatively flat surfaces. The reason for this difference may be that the slenderness ratio of the fibers and the fiber density of the layered fibers have the functional differences on different surfaces. By assembling independent vertical wedge-shaped microarrays and inclined cylindrical microarrays, the layered directional adhesive microstructures were also constructed [122]. Due to the addition of the inclined cylindrical microarrays, the layered directional adhesive microstructures not only have better adaptability to surfaces, but also can selfalign the terminal features, so that the load is evenly distributed to a single vertical wedge-shaped microstructure as much as possible. These improved performances increase the adhesive capacity of the layered directional adhesive microstructures on rough surfaces by five times, and also enable the layered directional adhesive microstructures to support a quadruped robot to achieve robust climbing on the semi-rough vertical surface. Compared with the vertical cylindrical microfibers, the mushroom-shaped microfibers have outstanding adhesive capacity due to their larger tip. Following the example of the double-layered vertical cylindrical microfibers, the researchers combined the multi-step micro-forming process and the dipping-transfer process to successfully prepare the double-layer mushroom-shaped microfibers to improve the adaptability of mushroomshaped microfibers to rough surfaces [123]. The indentation 
experiments confirmed that the double-layer mushroomshaped microfibers have higher flexibility and adhesive work, so it exhibits the stronger adhesive capacity under the same preload. However, their adhesive capacity on actual uneven or rough surfaces needs to be further explored.

Drawing lessons from the layered structure of gecko's foot to design artificial microfiber arrays with layered structure is a passive strategy to improve the adaptability of gecko-like dry adhesive surface on rough surfaces. In this design, the slenderness ratio of the fiber should be considered to make it flexible enough to easily adapt to the rough surface morphology and form conformal contact as much as possible; on the other hand, the modulus of the fiber should also be considered to make it hard enough not to collapse; in addition, it is necessary to weigh the relative size of the fiber geometry (especially the tip shape) and the rough surface topography, so that the adaptability of fiber to the rough surface matches the actual rough surface. After the fiber is designed, the corresponding processing technology needs to be further studied, which is often a challenge. It is precisely because the multi-scale layered fiber arrays are not easy to process, there are not many layered fibers appearing at present, so further research is needed.

In addition, a very small number of research teams tried another active strategy to improve the adaptability of geckolike dry adhesive surfaces on rough surfaces, namely integrating the electrostatic adhesion and gecko-like dry adhesion to make them work synergistically. The electrostatic adhesion originates from the electrostatic attraction induced by electrostatic induction or electric polarization between the conductive electrode and the substrate under the action of an electrostatic field, which can act on most substrate materials and surface morphologies. Combining the electrostatic adhesion and the gecko-like dry adhesion should be able to promote the overall adhesive capacity and improve their surface adaptability. The team led by Carlo Menon realized the integration of conductive electrodes and mushroom-shaped microstructures by embedding carbon black particles in the polymer, thus taking the lead in creating a dry adhesive surface with conductive mushroom-shaped microstructures [124]. The dry adhesive surface has conductivity due to the embedded conductive particles. As long as a high potential difference is applied between two independent dry adhesive surfaces, they can generate electrostatic adhesion on the non-conductive substrate. The results confirmed that due to the integration of electrostatic adhesion, the dry adhesive surface with conductive mushroom-shaped microstructure not only has the improved tangential adhesive strength, but also has the ability to self-preload. This selfpreloading capability enables the tip of mushroom-shaped microstructure to adapt to the surface morphology of the substrate to a greater extent, thereby increasing the contact area and ultimately enhancing the adhesion. Nevertheless, it cannot be directly used to adhering the conductive substrates because the entire conductive dry adhesive surface is completely exposed as a conductive electrode. To this end, the team further constructed independent comb-shaped electrodes based on conductive polymers and embedded them in a single dry adhesive surface with mushroom-shaped microstructures [125]. This improvement is not only conducive to the scaling design of the entire module, but also enables the electrostatic adhesion of the adhesive module to be applied on a conductive substrate.

In view of the advantages of integrating the electrostatic adhesion and the gecko-like adhesion, the independent coplanar concentric circular electrodes were also embedded into the gecko-like dry adhesive surface with vertical wedge-shaped microstructures [126]. The results showed that the electrostatic adhesion can promote the adhesive capacity of gecko-like dry adhesive surface with vertical wedge-shaped microstructures, and the promotion effect becomes more and more obvious with the increase of surface roughness of substrate. For further improving the adhesive capacity of the hybrid adhesive components, the research team further improved the entire hybrid adhesive components from the perspective of optimizing the electrostatic adhesion. Considering that the electrostatic adhesion is greatly affected by the electrode geometry, the double-layer concentric circular electrode was proposed [127]. The electrode was proven not only to have good flexibility, but also to increase the tangential adhesive capacity of the adhesive component by six times. Moreover, the relative permittivity of the dielectric layer was increased by incorporating highly polarized materials into the dielectric layer of the electrostatic adhesion with the double-layer comb-shaped electrodes. As a consequence, the tangential adhesive capacity of the electrostatic adhesion was increased by 4 times, and the tangential adhesive capacity of the hybrid adhesive component on the surface of different materials was increased by 2 times [128]. Recently, the coplanar comb electrodes were also embedded into the vertical cylindrical microfiber array [129]. Experimental confirmed that the addition of electrostatic adhesion not only improves the normal adhesive capacity of the hybrid component, but also reduces its need for net preload. In particular, when the supply voltage of electrostatic adhesion exceeds $1.5 \mathrm{kV}$, the compression preload required for the fiber adhesion can be provided by the electrostatic adhesion, which is extremely advantageous for picking up fragile targets.

In general, the introduction of the electrostatic adhesion not only improves the adhesive capacity and the surface adaptability of gecko-like adhesion, but also makes the hybrid adhesive component has the ability to self-preload. The promotion is due to the fact that the electrostatic adhesion is complementary to the gecko-like adhesion, which forms a positive feedback during the adhesion process: the electrostatic attraction (as a long-range force) can compensate for the preload during the preloading process to make the gecko-like adhesion better 
adapt to the surface and form a closer contact, which makes the Van der Waals force (as a short-range force) better activated; the enhancement of the Van der Waals force further reduces the gap between the electrostatic adhesive element and the substrate, so that the electrostatic attraction is improved.

\section{The Applications of Gecko-Like Dry Adhesive Surfaces}

The research goal of gecko-like dry adhesive surfaces is to reproduce the strong adhesive capacity and excellent adhesive characteristics of gecko's foot. A more comprehensive reproduction of the adhesive capacity and adhesive characteristics of gecko's foot is of great significance for the more effective application of gecko-like dry adhesive surfaces in practice. Although the layered microstructure of gecko's foot is difficult to replicate completely one-to-one at present, its excellent adhesive characteristics have been able to be partially reproduced, which greatly encourages researchers to explore related applications based on the gecko-like dry adhesive surfaces with limited adhesive capacity and characteristics. With the continuous renewal of gecko-like dry adhesive surfaces in recent years, its application potential has also been tapped. This section will summarize the application researches of gecko-like dry adhesive surfaces from three aspects: climbing robots, robot grippers and medical adhesive tapes.

\subsection{Climbing Robot}

The researches on the gecko-like dry adhesive surfaces are inspired by the adhesive system of gecko's foot, so it is more appropriate to apply the gecko-like dry adhesive surfaces directly to the field of climbing robots. Inspired by the gecko, the four-legged climbing robot called Stickybot (as shown in Fig. 8a) was developed, which uses the gecko-like dry adhesive surface based on directional polymer stalk as the adhesive unit of foot [130]. The climbing robot combines the multi-level flexibility of the overall structure and the directionality of the adhesive unit, and adopts the force feedback control strategy uniting a stiffness controller to successfully climb on a smooth vertical surface. Nevertheless, it can only climb upwards due to the insufficient degrees of freedom [131]. Moreover, to climb on a relatively rough surface and possess the stronger anti-fouling ability, an adhesive unit with a finer multi-level layered fiber structure should be adopted [122]. Considering that it is not easy for the robot to achieve accurate alignment between the adhesive unit and the substrate during the climbing process, the improved adhesive feet based on wedge-shaped microstructures were also proposed successively $[132,133]$. The improved adhesive feet simulate the macroscopic structure of the gecko's toes, and they cooperate with different supporting elements through tendons to preload the wedge-shaped microstructure, which not only maximizes the contact area between the wedge-shaped microstructure and the substrate, but also makes the load on the microstructure evenly distributed. Based on these improved adhesive feet, the climbing robots with a larger dead weight, such as Stickybot III (as shown in Fig. 8b) and Rise (as shown in Fig. 5c), also successfully climbed up on the vertical glass surface [132, 133]. Abigaille II, as a lightweight hexapod robot prototype (as shown in Fig. 8g), used the dry adhesive surface with double-layer mushroom-shaped microstructure and the pentapedal gait to climbing on a vertical resin surface [134]. Compared with the climbing robots of Stickybot series, Abigaille II has a completely symmetrical mechanical configuration and more degrees of freedom, which make it not only able to achieve all-round movement on the vertical surface, but also to realize the transfer from the horizontal surface to the vertical surface. Although Abigaille II has been optimized under quasi-static conditions, its actual climbing speed is slow, and the adhesive state of foot is easily destroyed by vibration during climbing. To this end, Abigaille III, as an improved version of Abigaille II, was developed with further optimized in the overall structure and control system (as shown in Fig. 8i) [135]. It also abandons the trajectory-based passive peeling mechanism used by Abigaille II and uses an independent motor to complete the active peeling of foot. Therefore, Abigaille III not only inherits the flexible climbing and transfer ability of Abigaille II on the vertical plane, but also has the ability to steadily climb on the uneven surfaces. Besides, Abigaille III was proven to be able to climb for $4 \mathrm{~h}$ and even wander for $7 \mathrm{~h}$. Although the mobility of climbing robots represented by Abigaille III on different inclined surfaces has been greatly improved, these legged robots do not have the ability to climb on an inverted ceiling currently. UNIclimb, as another developed gecko-like quadruped robot, takes the lead to make up for this shortcoming, namely it can crawl steadily on an inverted ceiling at a speed of $1 \mathrm{~mm} / \mathrm{s}$ [136]. The ability of the climbing robot to climb upside down benefits from the mushroom-shaped microstructure with strong adhesive capacity at the end of its feet and the used energy-efficient peeling method. The former provides a guarantee for its stable adhesion, while the latter lays the foundation for its easy peeling. Inspired by the fact that the gecko uses the tendon to flip their feet, an imitated gecko foot based on a mushroom-shaped microstructure was also designed [137]. The imitated gecko foot also achieves peeling through the turnover movement of foot driven by the tendon, which is similar to the adhesive foot of Stickybot. But the normal adhesive capacity of the imitated gecko foot does not depend on the preloading direction because of the isotropy of the mushroom-shaped microstructure, which allows it to assist a $700 \mathrm{~g}$ quadruped climbing robot (as 
Fig. 8 The climbing robots based on the different geckolike dry adhesive surfaces: a Stickybot [130]; b Stickybot III [132]; c Rise [133]; d LEMUR 3 [138]; e ACROBOT [141]; f the miniature climbing robot [142]; $\mathbf{g}$ Abigaille II [134]; $\mathbf{h}$ quadruped climbing robot with the imitated gecko foot [137]; i Abigaille III [135]; j TBCP-II [140]; k Waalbot II [139]

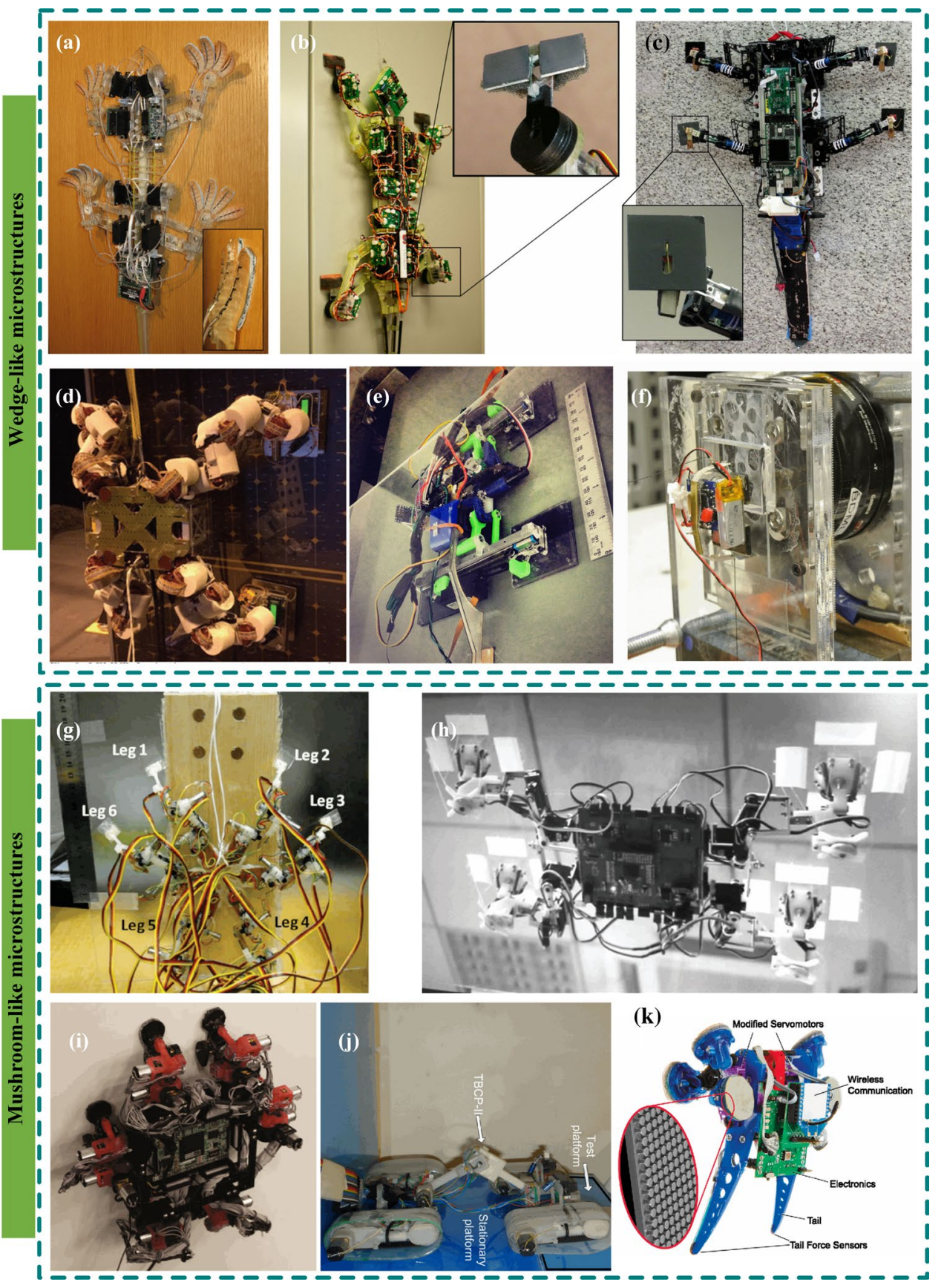

shown in Fig. 8h) to achieve a smooth inverted climb using a diagonal gait. The gripper constructed based on four pairs of opposed adhesive units was also used as the adhesive foot of the quadruped robot of LEMUR 3 (as shown in Fig. 8d), and the adhesive foot successfully enabled the robot to climb on the vertical solar windsurfing board [138].

Apart from the climbing robots based on legged movement, the gecko-like dry adhesive surfaces also play a role in climbing robots using other movement methods. Waalbot II is a wheel-legged climbing robot based on a dry adhesive surface with mushroom-shaped microstructures (as shown in
Fig. 8k) [139]. Due to its dexterous structure and control system, the climbing robot has the mobility and adaptability on different surface and terrain that far exceed the legged climbing robots. It can not only climb and transfer on surfaces with different directions, but also maneuver on an inverted smooth surface. Although Waalbot II has an adhesive recovery mechanism based on the swing maneuvering, it still does not have the ability to adapt to dirty outdoor environments. However, it can be improved using the gecko-like dry adhesive surfaces with self-cleaning capability in the future. The tracked robot has a simpler structure than the legged robot, 
and its climbing ability based on the gecko-like dry adhesive surface has also been confirmed. For example, based on the adhesion generated by the mushroom-shaped microstructure on the track, TBCP-II realized the climbing transfer from horizontal to vertical or from vertical to horizontal surface by actively adjusting the center height of the middle connecting joint of the module (as shown in Fig. 8j) [140]. In addition, the gecko-like dry adhesive surface was applied to a creeping climbing robot. ACROBOT can use two pairs of opposed adhesive pads to crawl on a vertical smooth surface with a load $(200 \mathrm{~g}$ ) at a creep speed of $0.15 \mathrm{~cm} / \mathrm{s}$ (as shown in Fig. 8e) [141]. The adhesive pad contains a flexible wedge-shaped microstructure with an inclined mushroomshaped tip, and the opposed configuration also makes the adhesive unit independent the direction of gravity. Therefore, the climbing robot can climb in any direction of gravity or even zero gravity, but this ability needs to be further studied. Also based on the inchworm locomotion gait, a miniature climbing robot using the controllable dry adhesive surface with a wedge-shaped microstructure as the adhesive foot was proposed (as shown in Fig. 8f) [142]. The robot cannot only climb on a vertical smooth surface at a speed of $3 \mathrm{~mm} / \mathrm{s}$, and it also has a load capacity of more than one kilogram. Thus, it is unmatched by all the aforementioned climbing robots. However, the maneuverability of this robot is not as good as other climbing robots because of the directionality of the adhesive foot. Recently, the gecko-like dry adhesive surface with vertical triangular micro-pillars was applied to the soft robot (Geca-Robot) which also uses the inchworm locomotion gait to crawl. The gecko-like dry adhesive surface, as the adhesive foot of Geca-Robot, not only greatly improves the terrain adaptability of the soft robot, but also enables the soft robot to carry objects with 50 times its own weight for crawling. Due to its light-induced motion mechanism, its crawling speed $(\sim 2.4 \mu \mathrm{m} / \mathrm{s})$ is quite slow.

Based on the above-related researches, it can be seen that there are no climbing robots using the gecko-like dry adhesive surface that can match the climbing ability of actual gecko. The reason for this situation stems from the limited adhesive capacity and characteristics of the existing gecko-like dry adhesive surface, and on the other hand, it also attributes to the fact that the body structure and control system of climbing robot are not as ingenious as the gecko at present. Climbing robots based on gecko-like dry adhesive surfaces are of great significance for applications such as monitoring, observation and even rescue. It is necessary to conduct further research on these issues in the future.

\subsection{Robot Grippers}

Compared with the conventional adhesive principles based on vacuum negative pressure adhesion and magnetic adhesion, the gecko-like dry adhesive surface based on the Van der Waals force adhesive mechanism not only sets rid of the dependence on the interface material, but also does not require additional energy to maintain adhesion. Especially for the gecko-like dry adhesive surface with controllable adhesive characteristic, it can easily complete the attachment and detachment for the target surface according to different control mechanisms. In this case, small grippers with different types of gecko-like dry adhesive surfaces as the adhesive elements were developed [81, 143, 144]. These grippers have been proven to be capable of robustly adhering and gripping lightweight and fragile targets such as silicon wafers, and be able to carry out the transfer operation for these targets. Among them, the three-legged gripper with the wedge-shaped microstructure arrays as the adhesive element has the most outstanding performance (as shown in Fig. 9a). This gripper uses a mechanism of shear induction instead of normal preload to activate the adhesive function of adhesive element, and can realize the adhering and grasping operation of silicon wafer in both non-vacuum and vacuum environment [81]. The ability of the three-legged gripper to hold silicon wafers steadily in a vacuum environment makes it possible to replace the electrostatic adhesive systems in the semiconductor industry. Adopting the same mechanism of shear induction, some three-legged grippers based on different transmission mechanisms for adhering larger scale planar targets were also proposed. In the gripper based on tendon transmission (as shown in Fig. 9b), the power transmitted by the load transfer pin is divided into three by the load distributor and is inputted to the three transmission tendons, and the transmission tendons then apply the input power in the form of shear load to the three adhesive pads distributed symmetrically on the circumference and radial direction, thereby exciting the adhesive pads to produce adhesion [145]. The research showed that the gripper can perform more than one hundred pick-and-place operations on glass or carbon fiber boards without no obvious adhesion degradation. Nevertheless, the gripper is still unable to fine apply the activation load, so that it cannot achieve the fine control of the adhesion. The slackness of the transmission tendon will also affect the synchronization of the three adhesive pads over time, thereby reducing the reliability and stability of the gripper. For this reason, two improvements to the gripper were proposed. Replacing the entire drive and transmission parts of the original gripper with the series-connected shape memory alloy wire not only greatly reduces the size of the gripper, but also improves the uniformity of the load on the adhesive pads. At the same time, it also enables the gripper to quantitatively apply tangential preload by adjusting the input voltage, thereby realizing quantitative control of normal adhesion [146]. Although the gripper based on shape memory alloy wire has comparable adhesive capacity to the original gripper, the high thermal hysteresis characteristic of the shape memory alloy wire significantly affects 


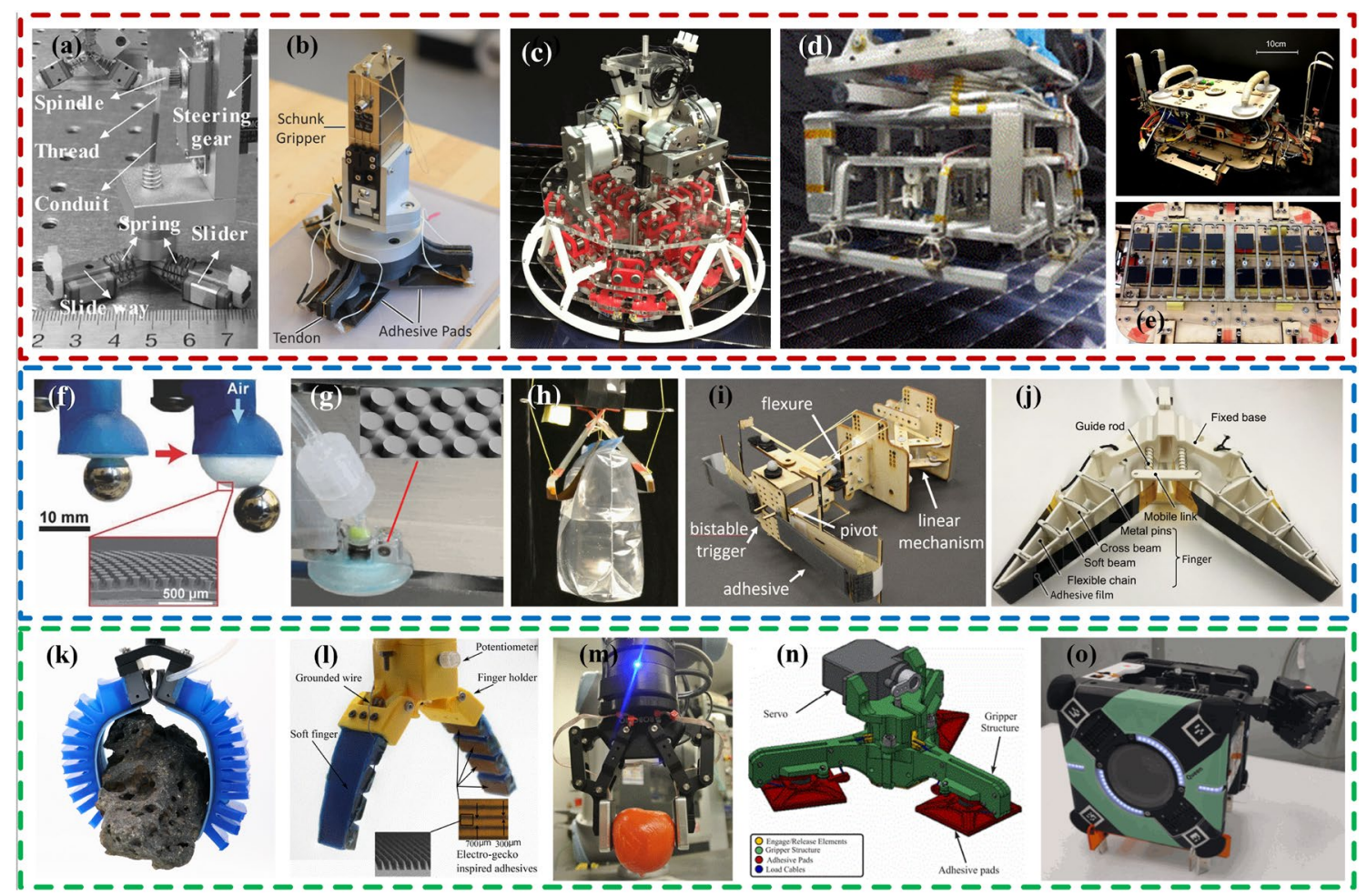

Fig. 9 The robot grippers based on the different gecko-like dry adhesive surfaces: a the three-legged gripper for adhering the smaller scale silicon wafers [81]; $\mathbf{b}$ the three-legged grippers based on tendon transmission for adhering larger scale planar targets [145]; $\mathbf{c}$ the gripper based on parallel constant force springs for adhering large space floating targets [153]; d the gripper based on differential pulleys for adhering large space floating targets [154]; e the robot gripper that integrates both two pairs of opposed curved surface adhesive unit based on tangential adhesion and eight pairs of opposed planar adhesive unit based on normal adhesion [160]; f GeckoGripper [148]; $\mathbf{g}$ inflatable soft gripper with a deformable support cavity [150]; $\mathbf{h}-\mathbf{j}$

the efficiency of the gripper in the pick-and-place operation, and it also has the same problem of slackness as the tendon. In view of the above, other solution was proposed, namely using three Scott-Russell mechanisms to replace tendons or shape memory alloy wires to transmit power. The solution not only makes the gripper more robust and reliable, but also improves the normal adhesive capacity of the gripper under certain preload conditions [147].

Although these grippers based on the three-legged design have certain advantages in resisting side loads, these grippers have higher requirements for the synchronization of the three adhesive pads. Moreover, the adhesive failure of any adhesive pad will results in the failure of the entire grasping task. In addition, these grippers only contain three adhesive pads, so their overall adhesive capacities are limited, and the backing layers of the adhesive pads are rigid. Thus, they are also limited to adhering and grasping flat targets. To make the gecko-like dry adhesive surface can be used to adhere and grasp a wider range of targets (such as non-planar the grippers based on tangential adhesion [156, 157, 159]; $\mathbf{k}$ the traditional pneumatic-driven soft robot gripper integrating the geckolike dry adhesive surface [166]; I the tendon-driven soft robot gripper integrating the gecko-like controllable adhesive surface [167]; $\mathbf{m}$ the parallel jaw gripper integrating a pair of wedge-shaped microstructure arrays arranged in the diagonal chevron pattern [168]; $\mathbf{n}$ the landing leg for assisting the aircraft to landing [164]; o the geckolike adhesive gripper for assisting the free-flying robot of Astrobee to complete temporary landing and grasping operations in the International Space Station [165]

targets), a few soft grippers integrated with the gecko-like dry adhesive surface were developed. GeckoGripper is an inflatable soft gripper that incorporates a mushroom-shaped microstructure array (as shown in Fig. 9f), which switches the adhesive state of the mushroom-shaped microstructure array by switching the expansion state of the inflatable film. When the gripper needs to release the target, the sufficient positive pressure is applied to expand the inflatable film, which causes the mushroom-shaped microstructure to be peeled off $[148,149]$. Because of the flexibility of the inflatable film on the GeckoGripper, the soft gripper has been proven to be able to pick-and-place parts of various geometric shapes (such as steel balls, wrenches and paper clips). However, its overall adhesive strength is also weakened. To make up as much as possible for the loss of the gripper's overall adhesive strength caused by the flexibility of the inflatable membrane, the rigid support cavity of the gripper was replaced by a deformable support cavity containing an array of flexible columns in the further research. As a 
result, the gripper can evenly distribute the adhesive load on the adhesive interface through the negative pressure difference, and obtain the improved adhesive strength without sacrificing the flexibility (as shown in Fig. 9g) [150]. For all that, the inflatable membrane can only adjust its deformation globally when the gripper adapts to the target, so the gripper is not easy to adapt to the local rough contour of the target surface. For this reason, the soft gripper based on the micro-zone control mechanism was studied to improve its ability to adapt to the rough surfaces [151]. The main difference between this gripper and the former is that its layer of mushroom-shaped microstructures is supported by the array of discrete vertical micro-pillars, and the locally discrete air chambers are formed between the micro-pillar arrays. In this case, the gripper can adjust the deformation of the adhesive layer in micro-regions through the deformation of local discrete air chambers, which further promotes the close contact between the microstructures and the rough surface. In addition to the above-mentioned soft grippers based on pneumatic control, the prototype of soft gripper based on electro-activation was proposed [152]. The switch of the adhesive state of the soft gripper is achieved by indirectly inducing the deformation of the mushroom-shaped microstructures by the heat generated by the electro-thermal film. Since its response speed is constrained by the thermal conductivity of the thermal module, the speed of pick-and-place operation is slow. Although the soft grippers based on the mushroom-shaped microstructures can adapt to a relatively wide range of targets, its adhering and grasping ability still needs to be further improved. For example, the dependence of their adhesive strength on the normal preload makes them difficult to adhering and grasping the space floating targets.

The mechanism of shear induction is a simulation of the adhesive control mechanism of gecko's foot. The grippers based on this mechanism can turn on its adhesive function and even adjust its adhesive capacity through the application of internal tangential load. In this case, they will not cause significant interference or even damage to the target surface, so they have great potential in adhering and grasping floating targets in space. To make the gripper based on the shear-induced mechanism capable of steadily adhering and gripping large space floating targets, some different technical solutions have been proposed. According to the additivity of Van der Waals force, the large adhesive capacity is required to adhere and grasp a large target, and the large adhesive capacity requires the large adhesive area. Due to the fact that the actual adhesive interface is not absolutely flat, the single large adhesive pad not only increases the difficulty of interface alignment, but could also make the adhesive capacity worse. For this reason, the strategy based on the arraying of multiple small adhesive units to form a large adhesive area was proposed, and two different load-sharing mechanisms were also proposed to make the distribution of adhesive load between the arrays more uniform. In the design based on parallel constant force springs (as shown in Fig. 9c), the loading tendons of each pair of opposed adhesive units are respectively connected to the constant force springs, so each adhesive unit undergoes loading independently during the loading process, and finally has approximately the same limit adhesion which is determined by the constant force spring [153]. In the design based on differential pulleys (as shown in Fig. 9d), the loading tendons of each pair of opposed adhesive units are respectively connected to the output ends of the differential pulleys, so that all the adhesive units experience the same loading load because of the synergetic movement of the output ends of the pulleys during the entire loading process [154]. Studies confirmed that the grippers based on the two load-sharing mechanisms can capture the local plane area of the floating target in space, and the gripper based on the parallel constant force spring is not sensitive to the adhesive failure of a single adhesive unit, while the gripper based on the differential pulleys is not sensitive to the difference of the target surface [154].

The above-mentioned grippers are only based on the normal adhesive capacity of the gecko-like controllable dry adhesive surface to complete the adhering and gripping task. Yet, for most gecko-like dry adhesive surfaces based on shear-induced mechanism to produce the controllable adhesive characteristic, their tangential adhesive capacities are generally stronger than the normal adhesive capacities. In the design of the robot gripper based on the gecko-like dry adhesive surface, ignoring the tangential adhesive capacity of the dry adhesive surface will be a loss. In this case, some grippers based on the tangential adhesive capacities of the dry adhesive surfaces were designed [155-159]. The kind of grippers based on the tangential adhesion generally include two symmetrically arranged flexible gecko-like dry adhesive surfaces (as shown in Fig. 9h-j), and their tangential adhesive capacities do not depend on the normal pressure. As a result, the kind of grippers can not only gently pickand-place fragile and deformable targets, but also adhere and grasp targets with large radius of curvature. Inspired by this, the robot gripper that integrates both the opposed curved surface adhesive unit based on tangential adhesion and the opposed planar adhesive unit based on normal adhesion was developed (as shown in Fig. 9e) [160]. The gripper contains eight pairs of opposed planar adhesive units arranged in a square array and two pairs of opposed curved surface adhesive units, all of which uniformly distribute the loads by the pulley system. In addition, the gripper integrates a nonlinear passive compliant wrist joint based on shape memory alloy wire, which has two levels of stiffness: the high stiffness enables the gripper to accurately operate the target, while the low stiffness makes it have the function of overload protection. Studies confirmed that whether it is to adhere and grasp a glass panel with flat features or an aluminum cylinder with 
curved features, the gripper has an absolute normal adhesive capacity of $65 \mathrm{~N}$, an absolute tangential adhesive capacity of $160 \mathrm{~N}$ and a corresponding torque resistance capacity of $6 \mathrm{~N} \cdot \mathrm{m}$. As a consequence, it has the potential to adhere and grasp large non-cooperative targets (such as space debris) in a microgravity environment.

The grippers based on the gecko-like dry adhesive surfaces have been not only intuitively used to adhere and grasp the target, but also tried as the landing leg of the small rotorcraft to assist the aircraft to temporarily land on a vertical wall or ceiling with less energy consumption and longer time of endurance [161-163]. To minimize the requirements for the dynamic maneuverability and initial impact velocity made by the opposed adhesive units during assisting the aircraft to landing, the landing leg similar to the three-legged gripper was designed (as shown in Fig. 9n) [164]. The landing leg only requires the impact velocity of $0.4 \mathrm{~m} / \mathrm{s}$ to ensure that the aircraft can safely land on a vertical smooth wall. The gripper based on the gecko-like adhesion for assisting the free-flying robot of Astrobee to complete temporary landing and grasping operations in the International Space Station was also proposed (as shown in Fig. 90) [165]. Since the adhesive capacity of the single opposed adhesive units is sufficient in a microgravity environment, the gripper is equipped with two pairs of independent opposed adhesive units for the purpose of adhesive redundancy. Currently, there are no reports about the gripper's performance on the International Space Station.

In addition to these independently designed grippers based on the gecko-like dry adhesive surfaces, the geckolike dry adhesive surfaces have been also used to assist in enhancing the gripping capabilities of existing grippers. Integrating the gecko-like dry adhesive surface on the surface of the traditional pneumatic-driven soft robot gripper not only enables the gripper to achieve high-intensity gripping at a lower pressure level, but also enables the gripper to have more gripping configurations (as shown in Fig. 9k) [166]. The soft robot gripper based on the driving tendon also has a greater gripping force due to the integration of the gecko-like controllable adhesive surface (as shown in Fig. 91) [167]. Furthermore, integrating a pair of wedgeshaped microstructure arrays arranged in the diagonal chevron pattern on the two fingertip surfaces of a parallel jaw gripper (as shown in Fig. 9m) not only enables the gripper to pick up the fragile targets (such as a rotten tomato) with extremely low gripping force, but also makes it have the ability to resist the moment from the non-coincidence between the grasping center and the center of gravity of the target [168]. The improvements of grasping ability of the gecko-like controllable adhesion to the traditional gripper are attributed to the strong tangential adhesion of gecko-like controllable dry adhesive surfaces. Although the tangential adhesion has a strong dependence on the loading direction, it does not depend on the large normal preload.

\subsection{Medical Adhesive Tapes}

In view of the excellent adhesive characteristics of the gecko-like dry adhesive surfaces, their potential as a medical adhesive tape has also been successively confirmed. Earlier, a gecko-like dry adhesive surface with vertical conical microstructure array was first used to adhere tissue surfaces [169]. The dry adhesive surface is made of polyacrylate, and its surface is deliberately coated with a layer of oxidized dextran to improve its adhesive capacity on wet tissues through covalent cross-linking. Studies have confirmed that the dry adhesive surface is not only biocompatible, but also biodegradable. Thus, it is expected to be used as a new type of tissue adhesive tape to assist medical procedures, such as replacing sutures to assist wound healing [169]. The typical difference from the conventional gecko-like dry adhesive surfaces is that the adhesive enhancement for the soft deformable tissue is achieved by reducing the density of microstructure, namely reducing the ratio of the tip diameter to the pitch can enhance the interface contact (as shown in Fig. 10a).

Subsequently, the gecko-like dry adhesive surface owning the mushroom-shaped microstructures was proved to have the ability to adhere the human skin, and its adhesive capacity on the skin mainly depends on the slenderness ratio of the microstructure (the optimized slenderness ratio is 3 ). In this case, it has the ability to become a new medical skin patch or as a fixing unit of body surface device for a health monitoring system (as shown in Fig. 10b) [170]. Compared with the traditional acrylic-based wet medical patches, the dry adhesive surface owning the mushroom-shaped microstructures can not only be used repeatedly on the skin, but also has good biocompatibility, and long-term adhesion will not cause adverse reactions such as skin redness and swelling. Due to the unique peeling mechanism, it will not cause pain during the process of attachment and detachment. Nevertheless, the adhesive capacity of the unmodified vertical mushroom-shaped microstructures on the skin (the maximum adhesion: $1.3 \mathrm{~N} / \mathrm{cm}^{2}$ ) needs to be improved as a result of the unique dual roughness morphology of the skin. For this reason, a two-step process combining the replication molding and inking process was used for the manufacture of vertical mushroom-shaped microstructures. In this case, the tip and base of the mushroom-shaped microstructures have different modulus: the high modulus of the base ensures the stability of the microstructure during the adhesive process, while the low modulus of the tip enhances the flexibility of the microstructure during the adhesive process [171]. The results confirmed that the mushroom-shaped microstructures with two-level modulus not only obtain enhanced adhesive 


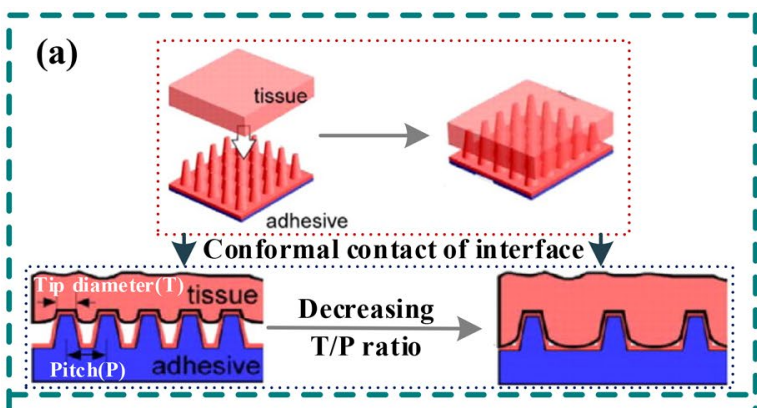

(c)
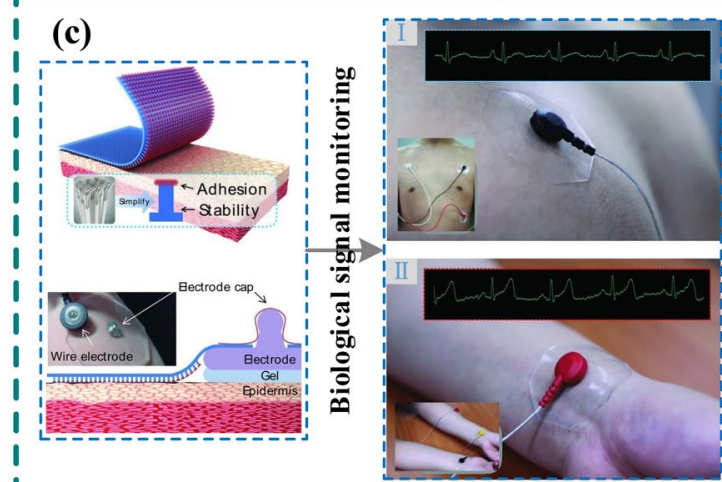

1 (e)
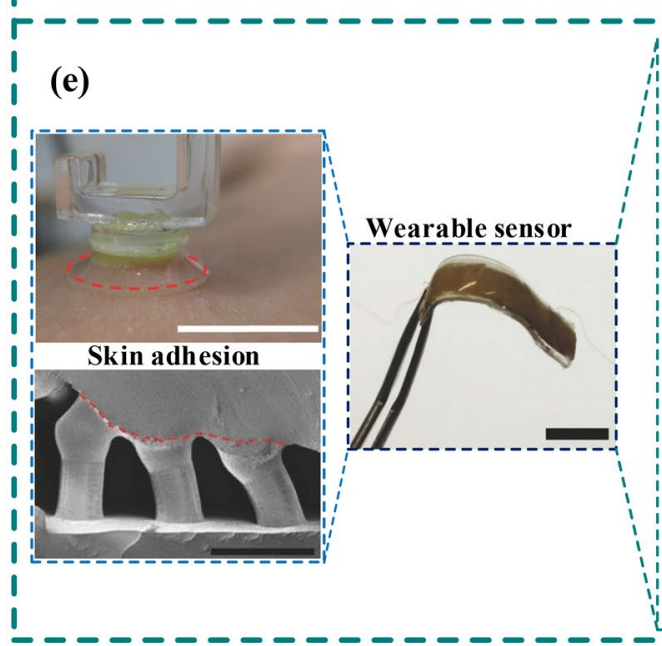

Fig. 10 The medical adhesive tapes based on the different gecko-like dry adhesive surfaces: a the gecko-like dry adhesive surface with vertical conical microstructure array was first used to adhere tissue surfaces [169]; b the gecko-like dry adhesive surface based on mushroom-shaped microstructures was regarded as the medical skin patch or as a fixing unit of body surface device for a health monitoring system [170]; c the mushroom-shaped microstructures with two-level modulus successfully assisted the electrodes of the health monitoring system to collect the ECG signals of the human chest and wrist [171];

capacity for skin surface (maximum adhesion: $2 \mathrm{~N} / \mathrm{cm}^{2}$ ), but also have higher durability. Based on the improved adhesive performance, the dry adhesive surface successfully assisted the electrodes of the health monitoring system to collect the ECG signals of the human chest and wrist, and it did not cause the irritation allergic reactions of skin during the continuous 48-h monitoring (as shown in Fig. 10c). Regrettably, (b)

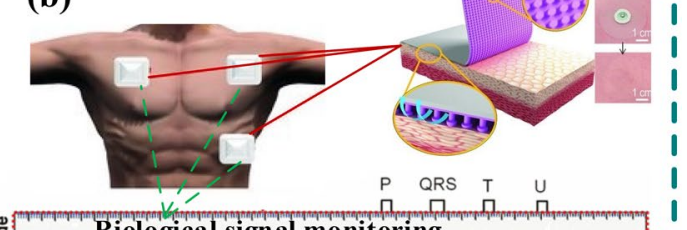

:

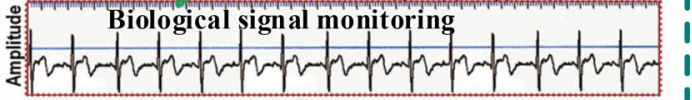

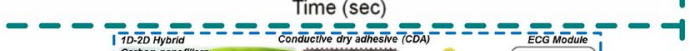

(d)
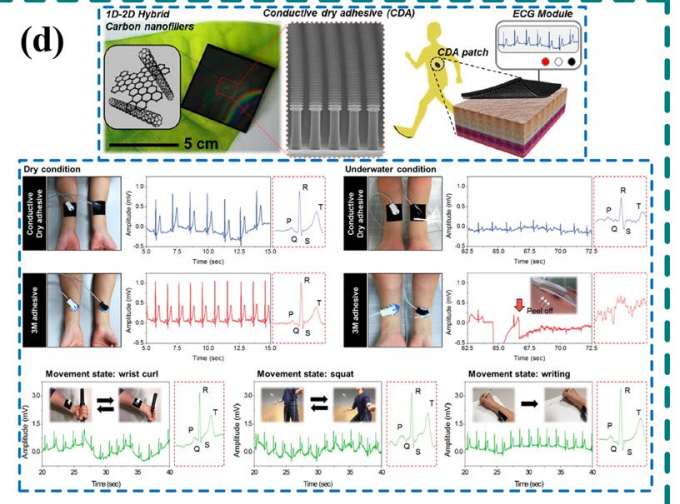

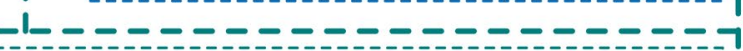
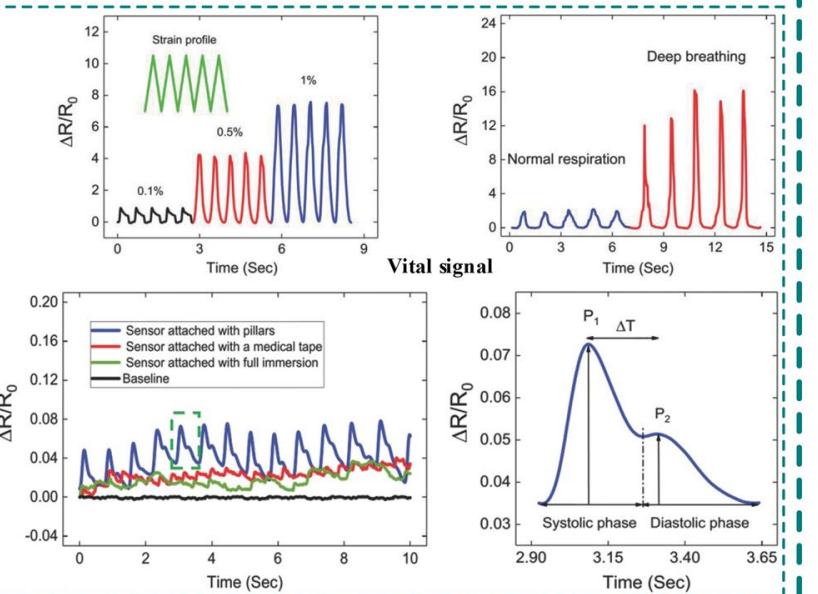

Time (Sec)

d the conductive dry adhesive surface mixed with carbon nanotubes and graphene was directly used for collecting the ECG signal of human body without integrating additional metal electrodes [172]; e the gecko-like dry adhesive surface replicating the layered morphology of the skin through the inking process can not only assist the flexible strain sensor to effectively monitor and record the human body's breathing, pulse and other vital signals, but also significantly improve the sensor's signal-to-noise ratio [173]

the metal electrode integrated on the dry adhesive surface can easily induce the dry adhesive surface to desorb around the electrode and extend to the entire dry adhesive surface. Therefore, it is currently limited to providing effective adhesion for the electrode when the human is in the relatively static state, such as sitting and sleeping. To solve the selfpeeling problem caused by the additional integrated metal 
electrode, the conductive dry adhesive surface mixed with carbon nanotubes and graphene was proposed [172]. The dry adhesive surface integrates the functions of skin adhesion and biological signal acquisition, which makes it can be directly used for collecting the ECG signal of human body without integrating additional metal electrodes. In addition, its own flexibility has not been inhibited because of its non-integration of metal electrodes, which makes it can be adhered on various curved skin surfaces. Even if the human body is in a certain state of motion, the dry adhesive surface can also maintain sufficient flexibility, thereby providing sufficient adhesion and achieve stable biological signal monitoring (as shown in Fig. 10d). More prominently, the conductive dry adhesive surface has been proven to be able to effectively collect the ECG signals containing medical information in an underwater environment, which is unmatched by traditional acrylic-based medical patches. The gecko-like dry adhesive surface that can make conformal contact with the surface of human skin was also proposed [173]. The gecko-like dry adhesive surface also included mushroom-shaped microstructures. The difference from other mushroom-shaped microstructures is that the tip of the mushroom-shaped microstructure is made of sticky vinyl siloxane, and its surface replicates the layered morphology of the skin through the inking process. As a result, it can form a conformal contact with the skin, thereby greatly improving its adhesive capacity to the skin (maximum adhesion: $1.8 \mathrm{~N} / \mathrm{cm}^{2}$ ). The research results confirmed that the dry adhesive surface can not only assist the flexible strain sensor to effectively monitor and record the human body's breathing, pulse and other vital signals, but also significantly improve the sensor's signal-to-noise ratio (as shown in Fig. 10e).

\section{Conclusion and Outlooks}

With the development of the mystery behind the gecko adhesion, the road of creating gecko-like dry adhesive surfaces has become increasingly lucid. Generally, the adhesive performances of gecko-like dry adhesive surfaces are affected by the characteristics of the matrix material and the geometry of the microstructure: the matrix materials with low modulus are more suitable for single-level microstructures, because they can make the ingle-level microstructures have good adaptability for surfaces only on account of their own flexibility; the matrix materials with high modulus are suitable for microstructures with high slenderness ratio and even multi-level microstructures, because their high rigidity is not conducive to the microstructures to adapt to the surfaces, which needs to be compensated by additional design of microstructure. Synthesizing diverse designs of microstructure, some special design experiences can be obtained: (1) making the microstructures with spatula-shaped, mushroom-shaped or funnel-shaped tip features can significantly enhance the adhesive strength of the gecko-like dry adhesive surfaces; (2) designing an anisotropic microstructure with inclined or even asymmetric features is the most direct and effective way to reproduce the controllable adhesive characteristics of gecko's foot; (3) designing a multi-scale compliant layered microstructure can improve the ability of the gecko-like dry adhesive surface to adapt to relatively rough surfaces. In addition to getting inspirations directly from the adhesive system of gecko's foot, some special strategies have also been proposed to improve the performances of the gecko-like dry adhesive surfaces: (1) using active stimulation methods such as temperature field, magnetic field, or electric field to change the deformation of the microstructure or the stiffness of backing layer of the microstructure can realize the switch control of the adhesive state of the gecko-like dry adhesive surfaces; (2) combining the electrostatic adhesion and the gecko-like dry adhesion can improve the overall adhesive strength and enhance the ability of the gecko-like dry adhesive surface to adapt to relatively rough surfaces; (3) integrating the electrostatic and ultrasonic self-cleaning function can significantly improve the self-cleaning efficiency of the gecko-like dry adhesive surfaces [176].

Currently, the gecko-like dry adhesive surfaces with mushroom-shaped microstructure or wedge-shaped microstructure have become an outstanding representative of gecko-like dry adhesive surfaces. They have been applied to the fields of climbing robots, robot grippers and even medical adhesive tapes according to the different adhesive performance. Nevertheless, there are still some problems to be solved in the research of gecko-like dry adhesive surfaces. First, it is necessary to further explore the strategies that can fabricate the multi-scale compliant layered microstructures like the gecko's foot. The adaptability of the gecko-like dry adhesive surfaces to the rough surfaces still needs to be improved at present, and the rough surfaces can easily cause the adhesive capacity of the gecko-like dry adhesive surfaces to decrease or even fail. Designing the multi-scale compliant layered microstructure is the most direct means to improve its adaptability to the rough surfaces. Second, there is still a lack of a complete adhesive model that simultaneously considers the material nonlinearity, the geometric nonlinearity, and the nonlinearity of interface interaction. The effective design of the gecko-like dry adhesive surface requires the support of the adhesive model behind it, but the current adhesive model describing the adhesive process of the gecko-like dry adhesive surface is not perfect as most of the current models use the simplified assumptions. In particular, no model can well couple the relationship of frictional adhesion presented by the gecko's foot, which makes it 
impossible to achieve a more complete optimization design. Third, further exploring the biological evolution mechanism behind the foot of geckos is also necessary. The current engineering methods still cannot integrate all the adhesive characteristics of gecko's foot into one product, so learning from the mechanism of nature's evolutionary design may be a solution. Finally, the related application researches based on the existing gecko-like dry adhesive surfaces should be further followed up, and the shortcomings in practical application will further promote the improvement and optimization of gecko-like dry adhesive surface. In any case, it will still be a long-term challenge to develop a gecko-like dry adhesive surface with wider environmental adaptability.

Acknowledgements This work was supported by the Major Research Plan of the National Natural Science Foundation of China [Grant number 91848202]. This work was also supported by the Self-Planned Task of State Key Laboratory of Robotics and System (HIT) [Grant number SKLRS202106B].

\section{Declarations}

Conflict of Interest The authors declare that they have no conflict of interest.

Open Access This article is licensed under a Creative Commons Attribution 4.0 International License, which permits use, sharing, adaptation, distribution and reproduction in any medium or format, as long as you give appropriate credit to the original author(s) and the source, provide a link to the Creative Commons licence, and indicate if changes were made. The images or other third party material in this article are included in the article's Creative Commons licence, unless indicated otherwise in a credit line to the material. If material is not included in the article's Creative Commons licence and your intended use is not permitted by statutory regulation or exceeds the permitted use, you will need to obtain permission directly from the copyright holder. To view a copy of this licence, visit http://creativecommons.org/licenses/by/4.0/.

\section{References}

1. Kwak, J. S., \& Kim, T. W. (2010). A review of adhesion and friction models for gecko feet. International Journal of Precision Engineering and Manufacturing, 11, 171-186.

2. Zhou, M., Pesika, N., Zeng, H. B., \& Israelachvili, J. (2013). Recent advances in gecko adhesion and friction mechanisms and development of gecko-inspired dry adhesive surfaces. Friction, $1,114-129$.

3. Li, Y., Krahn, J., \& Menon, C. (2016). Bioinspired dry adhesive materials and their application in robotics: A review. Journal of Bionic Engineering, 13, 181-199.

4. Ruibal, R., \& Ernst, V. (1965). The structure of the digital setae of lizards. Journal of Morphology, 117, 271-293.

5. Rizzo, N. W., Gardner, K. H., Walls, D. J., Keiper-Hrynko, N. M., Ganzke, T. S., \& Hallahan, D. L. (2006). Characterization of the structure and composition of gecko adhesive setae. Journal of the Royal Society Interface, 3, 441-451.

6. Alibardi, L. (2003). Ultrastructural autoradiographic and immunocytochemical analysis of setae formation and keratinization in the digital pads of the gecko Hemidactylus turcicus (Gekkonidae, Reptilia). Tissue \& Cell, 35, 288-296.

7. Peattie, A. M., Majidi, C., Corder, A., \& Full, R. J. (2007). Ancestrally high elastic modulus of gecko setal $\beta$-keratin. Journal of the Royal Society Interface, 4, 1071-1076.

8. Autumn, K., Majidi, C., Groff, R. E., Groff, R. E., Dittmore, A., \& Fearing, R. (2006). Effective elastic modulus of isolated gecko setal arrays. Journal of Experimental Biology, 209, 3558-3568.

9. Autumn, K., \& Peattie, A. M. (2002). Mechanisms of adhesion in geckos. Integrative \& Comparative Biology, 42, 1081-1090.

10. Chui, B. W., Kenny, T. W., Mamin, H. J., Mamin, H. J., Terris, B. D., \& Rugar, D. (1998). Independent detection of vertical and lateral forces with a sidewall-implanted dual-axis piezoresistive cantilever. Applied Physics Letters, 72, 1388-1390.

11. Autumn, K., Liang, Y. A., Hsieh, S. T., Zesch, W., Chan, W. P., Kenny, T. W., Fearing, R., \& Full, R. J. (2000). Adhesive force of a single gecko foot hair. Nature, 405, 681-685.

12. Autumn, K., Sitti, M., \& Liang, A. Y. (2002). Evidence for van der Waals adhesion in gecko setae. Proceedings of the National Academy of Science of the United States of America, 99, 12252-12256.

13. Huber, G., Mantz, H., Spolenak, R., Mecke, K., Jacobs, K., Gorb, S. N., \& Arzt, E. (2005). Evidence for capillarity contributions to gecko adhesion form single spatula nanomechanical mechasurements. Proceedings of the National Academy of Science of the United States of America, 102, 16293-16296.

14. Kim, T. W., \& Bhushan, B. (2008). The adhesion model considering capillarity for gecko attachment system. Journal of the Royal Society Interface, 5, 319-327.

15. Puthoff, J. B., Prowse, M. S., Wilkinson, M., \& Autumn, K. (2010). Changes in materials properties explain the effects of humidity on gecko adhesion. Journal of Experimental Biology, 213, 3699-3704.

16. Hill, G. C., Soto, D. R., Peattie, A. M., Full, R. J., \& Kenny, T. W. (2011). Orientation angle and the adhesion of single gecko setae. Journal of the Royal Society Interface, 8, 926-933.

17. Autumn, K., Hsieh, S. T., Dudek, D. M., Chen, J., Chitaphan, C., \& Full, R. J. (2006). Dynamics of gecko running vertically. Journal of Experimental Biology, 209, 260-272.

18. Autumn, K., Dittmore, A., Santos, D., Spenko, M., \& Cutkosky, M. R. (2006). Frictional adhesion: A new angle on gecko attachment. Journal of Experimental Biology, 209, 3569-3579.

19. Autumn, K. (2007). Gecko adhesion: Structure, function, and applications. Mrs Bulletin, 32, 473-478.

20. Zhao, B. X., Pesika, N., Rosenberg, K., Tian, Y., Zeng, H. B., McGuiggan, P., Autumn, K., \& Israelachvili, J. (2008). Adhesion and friction force coupling of gecko setal arrays: Implications for structured adhesive surfaces. Langmuir: the ACS Journal of Surfaces \& Colloids, 24, 1517-1524.

21. Zhao, B. X., Pesika, N., Zeng, H. B., Wei, Z., Chen, Y., Autumn, K., Turner, K., \& Israelachvili, J. (2009). Role of tilted adhesion fibrils (Setae) in the adhesion and locomotion of gecko-like systems. Journal of Physical Chemistry B, 113, $3615-3621$.

22. Wan, J., Tian, Y., Zhou, M., Zhang, X. J., \& Meng, Y. G. (2012) Experimental research of load effect on the anisotropic friction behaviors of gecko seta array. Acta Physica Sinica, 61, 016202.

23. Tian, Y., Tao, D. S., Pesika, N., Wan, J., Meng, Y. G., \& Zhang, X. J. (2015). Flexible control and coupling of adhesion and friction of gecko setal array during sliding. Tribology Online, 10, 106-114.

24. Tao, D. S., Wan, J., Pesika, N. S., Zeng, H. B., Liu, Z. Y., Zhang, X. J., Meng, Y. G., \& Tian, Y. (2015). Adhesion and friction of an isolated gecko setal array: The effects of substrates and relative humidity. Biosurface and Biotribology, 1, 42-49. 
25. Wang, Z. Y., Gu, W. H., Wu, Q., Ji, A. H., \& Dai, Z. D. (2010). Morphology and reaction force of toes of geckos freely moving on ceilings and walls. Science China Technological Sciences, 53, 1688-1693.

26. Parness, A., Soto, D., Esparza, N., Gravish, N., Wilkinson, M., Autumn, K., \& Cutkosky, M. (2009). A microfabricated wedgeshaped adhesive array displaying gecko-like dynamic adhesion, directionality and long lifetime. Journal of the Royal Society Interface, 6, 1223-1232.

27. Gravish, N., Wilkinson, M., \& Sponberg, S. (2010). Rate-dependent frictional adhesion in natural and synthetic gecko setae. Journal of the Royal Society Interface, 7, 259-269.

28. Hansen, W. R., \& Autumn, K. (2005). Evidence for self-cleaning in gecko setae. Proceedings of the National Academy of Science of the United States of America, 102, 385-389.

29. Hu, S. H., Lopez, S., Niewiarowski, P. H., \& Xia, Z. H. (2012). Dynamic self-cleaning in gecko setae via digital hyperextension. Journal of the Royal Society Interface, 9, 2781-2790.

30. Xu, Q., Wan, Y. Y., Hu, T. S., Liu, T. X., Tao, D. S., Niewiarowski, P. H., Tian, Y., Liu, Y., Dai, L. M., Yang, Y. Q., \& Xia, Z. H. (2015). Robust self-cleaning and micromanipulation capabilities of gecko spatula and their bio-mimics. Nature Communications, 6, 8949 .

31. Autumn, K., \& Hansen, W. (2006). Ultrahydrophobicity indicates a non-adhesive default state in gecko setae. Journal of Comparative Physiology A, 192, 1205-1212.

32. Autumn, K., Niewiarowski, P. H., \& Puthoff, J. B. (2014). Gecko adhesion as a model system for integrative biology, interdisciplinary science, and bioinspired engineering. Annual Review of Ecology Evolution \& Systematics, 45, 445-470.

33. Jagota, A., \& Bennison, S. J. (2002). Mechanics of adhesion through a fibrillar microstructure. Integrative \& Comparative Biology, 42, 1140-1145.

34. Arzt, E., Gorb, S., \& Spolenak, R. (2003). From micro to nano contacts in biological attachment devices. Proceedings of the National Academy of Sciences of the United States of America, 100, 10603-10606.

35. Sitti, M., \& Fearing, R. S. (2003). Synthetic gecko foot-hair micro/nano-structures for future wall-climbing robots. Journal of Adhesion Science and Technology, 17, 1055-1073.

36. Shah, G. J. \& Sitti, M. (2004). Modeling and design of biomimetic adhesives inspired by gecko foot-hairs. Proceedings of the 2004 IEEE International Conference on Robotics and Biomimetics, Shengyang, China, 873-878.

37. Gao, H. J., Wang, X., Yao, H. M., Gorb, S., \& Arzt, E. (2005). Mechanics of hierarchical adhesion structures of geckos. Mechanics of Materials, 37, 275-285.

38. Wang, H. J., Mei, T., Wang, X. H., Lin, X. M. (2005). Interaction and simulation analysis between the biomimetic gecko adhesion array and rough surface. Proceedings of the IEEE International Conference on Mechatronics \& Automation, Niagara Falls, Canada, 1063-1068.

39. Bhushan, B., Peressadko, A. G., \& Kim, T. W. (2006). Adhesion analysis of two-level hierarchical morphology in natural attachment systems for 'smart adhesion.' Journal of Adhesion Science and Technology, 20, 1475-1491.

40. Takahashi, K., Berengueres, J. O. L., Obata, K. J., \& Saito, S. (2006). Geckos' foot hair structure and their ability to hang from rough surfaces and move quickly. International Journal of Adhesion \& Adhesives, 26, 639-643.

41. Ren, N. F., Wang, X. H., Mei, T., Wang, H, J. (2006). Detachment control analysis for nanofabricated adhesive mimicking gecko foot-hairs. Proceedings of the 2006 IEEE International Conference on Mechatronics and Automation, Luoyang, China, 267-271.
42. Kim, T. W., \& Bhushan, B. (2007). Effect of stiffness of multilevel hierarchical attachment system on adhesion enhancement. Ultramicroscopy, 107, 902-912.

43. Gorb, S., Varenberg, M., Peressadko, A., \& Tuma, J. (2007). Biomimetic mushroom-shaped fibrillary adhesive microstructure. Journal of the Royal Society Interface, 4, 271-275.

44. Carbone, G., Pierro, E., \& Gorb, S. N. (2011). Origin of the superior adhesive performance of mushroom-shaped microstructured surfaces. Soft Matter, 7, 5545.

45. Kizikan, E., \& Gorb, S. N. (2018). Bioinspired further enhanced dry adhesive by the combined effect of the microstructure and surface free energy increase. ACS Applied Materials \& Interfaces, 10, 26752-26758.

46. Kendall, K. (1971). The adhesion and surface energy of elastic solids. Journal of Physics D-Applied Physics, 4, 1186-1195.

47. Kendall, K. (1975). Thin-film peeling-the elastic term. Journal of Physics D-Applied Physics, 8, 1449-1452.

48. Kendall, K. (1978). Interfacial dislocations spontaneously created by peeling. Journal of Physics D-Applied Physics, 11, 1519-1527.

49. Tian, Y., Pesika, N., \& Zeng, H. B. (2006). Adhesion and friction in gecko toe attachment and detachment. Proceedings of the National Academy of Sciences of the United States of America, 103, 19320-19325.

50. Pesika, N. S., Tian, Y., Zhao, B., Rosenberg, K., Zeng, H., Mcguiggan, P., Autumn, K., \& Israelachvili, J. N. (2007). Peel zone model of tape peeling based on the gecko adhesive system. Journal of Adhesion, 83, 383-401.

51. Chen, B., Wu, P. D., \& Gao, H. J. (2009). Pre-tension generates strongly reversible adhesion of a spatula pad on substrate. Journal of the Royal Society Interface, 6, 529-537.

52. Peng, Z. L., Chen, S. H., \& Soh, A. K. (2010). Peeling behavior of a bio-inspired nano-film on a substrate. International Journal of Solids and Structures, 47, 1952-1960.

53. Cheng, Q. H., Chen, B., Gao, H. J., \& Zhang, Y. W. (2012). Sliding-induced non-uniform pre-tension governs robust and reversible adhesion: A revisit of adhesion mechanisms of geckos. Journal of the Royal Society Interface, 9, 283-291.

54. Sauer, R. A., \& Holl, M. (2013). A detailed 3D finite element analysis of the peeling behavior of a gecko spatula. Computer Methods in Biomechanics \& Biomedical Engineering, 16, 577-591.

55. Sauer, R. A., \& Mergel, J. C. (2014). A geometrically exact finite beam element formulation for thin film adhesion and debonding. Finite Elements in Analysis and Design, 86, 120-135.

56. Mergel, J. C., Sauer, R. A., \& Saxena, A. (2014). Computational optimization of adhesive microstructures based on a nonlinear beam formulation. Structural and Multidisciplinary Optimization, 50, 1001-1017.

57. Mergel, J. C., \& Sauer, R. A. (2014). On the optimum shape of thin adhesive strips for various peeling directions. Journal of Adhesion, 90, 526-544.

58. Mergel, J. C., Sahli, R., Scheibert, J., \& Sauer, R. A. (2019). Continuum contact models for coupled adhesion and friction. Journal of Adhesion, 95, 1101-1133.

59. Gouravaraju, S., Sauer, R. A., \& Gautam, S. S. (2021). Investigating the normal and tangential peeling behavior of gecko spatula using a coupled adhesion-friction model. The Journal of Adhesion, 97, 952-983.

60. Gouravaraju, S., Sauer, R. A., \& Gautam, S. S. (2020). On the presence of a critical detachment angle in gecko spatula peeling-a numerical investigation using an adhesive friction model. The Journal of Adhesion. https://doi.org/10.1080/00218464. 2020.1746652 (in press).

61. Mergel, J. C., Scheibert, J., \& Sauer, R. A. (2021). Contact with coupled adhesion and friction: Computational framework, 
applications, and new insights. Journal of the Mechanics and Physics of Solids, 146, 104194.

62. Santos, D., Spenko, M., Parness, A., Kim, S., \& Cutkosky, M. (2007). Directional adhesion for climbing: Theoretical and practical considerations. Journal of Adhesion Science and Technology, 21, 1317-1341.

63. Filippov, A., Popov, V., \& Gorb, S. N. (2011). Shear induced adhesion: Contact mechanics of biological spatula-like attachment devices. Journal of Theoretical Biology, 276, 126-131.

64. Jeong, H. E., Lee, J. K., Kim, H. N., Moon, S. H., \& Suh, K. Y. (2009). A nontransferring dry adhesive with hierarchical polymer nanohairs. Proceedings of the National Academy of Sciences of the United States of America, 106, 5639-5644.

65. Aksak, B., Murphy, M. P., \& Sitti, M. (2007). Adhesion of biologically inspired vertical and angled polymer microfiber arrays. Langmuir, 23, 3322-3332.

66. Santos, D., Kim, S., Spenko, M., Parness, A., \& Cutkosky, M. (2007). Directional adhesive structures for controlled climbing on smooth vertical surfaces. Proceedings of the 2007 IEEE International Conference on Robotics \& Automation, Rome, Italy, 1262-1267.

67. Campo, A. D., Greiner, C., Álvarez, I., \& Arzt, E. (2007). Patterned surfaces with pillars with controlled 3D tip geometry mimicking bioattachment devices. Advanced Materials, 19, 1973-1977.

68. Varenberg, M., \& Gorb, S. (2007). Shearing of fibrillary adhesive microstructure: Friction and shear-related changes in pull-off force. Journal of the Royal Society Interface, 4, 721-725.

69. Kim, S., Aksak, B., \& Sitti, M. (2007). Enhanced friction of elastomer microfiber adhesives with spatulate tips. Applied Physics Letters, 91, 221913.

70. Murphy, M. P., Aksak, B., \& Sitti, M. (2007). Adhesion and anisotropic friction enhancements of angled heterogeneous microfiber arrays with spherical and spatula tips. Journal of Adhesion Science and Technology, 21, 1281-1296.

71. Aksak, B., Murphy, M.P., \& Sitti, M. (2008). Gecko inspired micro-fibrillar adhesives for wall climbing robots on micro/ nanoscale rough surfaces. Proceedings of the 2008 IEEE International Conference on Robotics and Automation, Pasadena, USA, 3058-3063.

72. Murphy, M. P., Aksak, B., \& Sitti, M. (2009). Gecko-inspired directional and controllable adhesion. Small (Weinheim an der Bergstrasse, Germany), 5, 170-175.

73. Wang, Z. Z., Gu, P., \& Wu, X. P. (2014). Gecko-inspired bidirectional double-sided adhesives. Soft Matter, 10, 3301-3310.

74. Wang, Y., Li, X. M., Tian, H. M., Hu, H., Tian, Y., Shao, J. Y., \& Ding, Y. C. (2015). Rectangle-capped and tilted micropillar array for enhanced anisotropic anti-shearing in biomimetic adhesion. Journal of the Royal Society Interface, 12, 20150090.

75. Kwak, M. K., Jeong, H. E., Bae, W. G., Jung, H. S., \& Suh, K. Y. (2011). Anisotropic adhesion properties of triangular-tip-shaped micropillars. Small (Weinheim an der Bergstrasse, Germany), 7, 2296-2300.

76. Seo, S., Lee, J., Kim, K. S., Ko, K. H., \& Lee, J. (2014). Anisotropic adhesion of micropillars with spatula pads. ACS Applied Materials \& Interfaces, 6, 1345-1350.

77. Wang, Y., Tian, H. M., Shao, J. Y., Sameoto, D., Li, X. M., Wang, L., Hu, H., Ding, Y. C., \& Lu, B. H. (2016). Switchable dry adhesion with step-like micropillars and controllable interfacial contact. ACS Applied Materials \& Interfaces, 8, 10029-10037.

78. Seo, J., Eisenhaure, J., \& Kim, S. (2016). Micro-wedge array surface of a shape memory polymer as a reversible dry adhesive. Extreme Mechanics Letters, 9, 207-214.

79. Day, P., Eason, E. V., Esparza, N., Christensen, D., \& Cutkosky, M. (2013). Microwedge machining for the manufacture of directional dry adhesives. Journal of Micro and Nano-Manufacturing, 1, 011001.

80. Kerst, C., Suresh, S. A., \& Cutkosky, M. R. (2020). Creating metal molds for directional gecko-inspired adhesives. Journal of Micro and Nano-Manufacturing, 8, 011004.

81. Tao, D. S., Gao, X., Lu, H. Y., Liu, Z. Y., Li, Y., Tong, H., Pesika, N., Meng, Y. G., \& Tian, Y. (2017). Controllable anisotropic dry adhesion in vacuum: Gecko inspired wedged surface fabricated with ultraprecision diamond cutting. Advanced Functional Materials, 27, 1606576 .

82. Wang, W., \& Xie, Z. W. (2021). Fabrication of a biomimetic controllable adhesive surface by ultraprecision multistep and layered scribing and casting molding. Science China Technological Sciences. https://doi.org/10.1007/s11431-020-1801-9 (in press).

83. Chary, S., Tamelier, J., \& Turner, K. (2013). A microfabricated gecko-inspired controllable and reusable dry adhesive. Smart Materials and Structures, 22, 025013.

84. Jin, K. J., Tian, Y., Erickson, J. S., Puthoff, J., Autumn, K., \& Pesika, N. S. (2012). Design and fabrication of gecko-inspired adhesives. Langmuir, 28, 5737-5742.

85. Jin, K. J., Cremaldi, J. C., Erickson, J. S., Tian, Y., Israelachvili, J. N., \& Pesika, N. S. (2014). Biomimetic bidirectional switchable adhesive inspired by the gecko. Advanced Functional Materials, 24, 574-579.

86. Reddy, S., Arzt, E., \& Campo, A. D. (2007). Bioinspired surfaces with switchable adhesion. Advanced Materials, 19, 3833-3837.

87. Chen, C. M., Chiang, C. L., Lai, C. L., Xie, T., \& Yang, S. (2013). Bucking-based strong dry adhesives via interlocking. Advanced Functional Materials, 23, 3813-3823.

88. Northen, M. T., Greiner, C., Arzt, E., \& Turner, K. L. (2008). A gecko-inspired reversible adhesive. Advanced Materials, 20, 3905-3909.

89. Drotlef, D. M., Blümler, P., \& Campo, A. D. (2014). Magnetically actuated patterns for bioinspired reversible adhesion (dry and wet). Advanced Materials, 26, 775-779.

90. Jiang, W. T., Wang, L. L., Liu, H. Z., Ma, H. Y., Tian, H. M., Chen, B. D., Shi, Y. S., Yin, L., \& Ding, Y. C. (2014). Bioinspired directional high-aspect-ratio nanopillars: Fabrication and actuation. RSC Advances, 4, 420002-442008.

91. Tan, D., Wang, X., Liu, Q., Shi, K., Yang, B. S., Liu, S., Wu, Z. S., \& Xue, L. J. (2019). Switchable adhesion of micropillar adhesive on rough surfaces. Small (Weinheim an der Bergstrasse, Germany), 15, 1904248.

92. Kim, S., Sitti, M., Hui, C. Y., Long, R., \& Jagota, A. (2007). Effect of backing layer thickness on adhesion of single-level elastomer fiber arrays. Applied Physics Letters, 91, 161905.

93. Long, R., Hui, C. Y., Kim, S., \& Sitti, M. (2008). Modeling the soft backing layer thickness effect on adhesion of elastic microfiber arrays. Journal of Applied Physics, 104, 044301.

94. Krahn, J., Sameoto, D., \& Menon, C. (2011). Controllable biomimetic adhesion using embedded phase change material. Smart Materials and Structures, 20, 015014.

95. Li, S., Tian, H. M., Shao, J. Y., Liu, H. R., Wang, D. R., \& Zhang, W. T. (2020). Switchable adhesion for nonflat surfaces mimicking geckos' adhesive structures and toe muscles. ACS Applied Materials \& Interfaces, 12, 39745-39755.

96. Krahn, J., Bovero, E., \& Menon, C. (2015). Magnetic field switchable dry adhesives. ACS Applied Materials \& Interfaces, 7, 2214-2222.

97. Sethi, S., Ge, L. H., Ci, L. J., Ajayan, P. M., \& Dhinojwala, A. (2008). Gecko-inspired carbon nanotube-based self-cleaning adhesives. Nano Letters, 8, 822-825.

98. Kim, S., Cheung, E., \& Sitti, M. (2009). Wet self-cleaning of biologically inspired elastomer mushroom shaped microfibrillar adhesives. Langmuir, 25, 7196-7199. 
99. Lee, J., \& Fearing, R. S. (2012). Wet self-cleaning of superhydrophobic microfiber adhesives formed from high density polyethylene. Langmuir, 28, 15372-15377.

100. Zhang, Y. C., Qu, S. X., Cheng, X., Gao, X. L., \& Guo, X. (2016). Fabrication and characterization of gecko-inspired dry adhesion, superhydrophobicity and wet self-cleaning surfaces. Journal of Bionic Engineering, 13, 132-142.

101. Lee, J., \& Fearing, R. S. (2008). Contact self-cleaning of synthetic gecko adhesive from polymer microfibers. Langmuir, 24, 10587-10591.

102. Gillies, A. G., Puthoff, J., Cohen, M. J., Autumn, K., \& Fearing, R. S. (2013). Dry self-cleaning properties of hard and soft fibrillary structures. ACS Applied Materials \& Interfaces, 5, 6081-6088.

103. Menguc, Y., Rohrig, M., Abusomwan, U., Holscher, H., \& Sitti, M. (2014). Staying sticky: Contact self-cleaning of geckoinspired adhesives. Journal of the Royal Society Interface, 11, 20131205 .

104. Abusomwan, U. A., \& Sitti, M. (2014). Mechanics of loaddrag-unload contact cleaning of gecko-inspired fibrillary adhesives. Langmuir, 30, 11913-11918.

105. Alizadehyazdi, V., Simaite, A., \& Spenko, M. (2019). Evaluation of material properties for practical microstructured adhesives: Low dust adhesion and high shear strength. ACS Applied Materials \& Interfaces, 11, 8654-8666.

106. Wan, Y. Y., \& Xia, Z. H. (2018). Self-cleaning and controlled adhesion of gecko feet and their bioinspired micromanipulators. MRS Advances, 3, 1641-1646.

107. Alizadehyazdi, V., Modabberifar, M., Akherat, S. M. J. M., \& Spenko, M. (2018). Electrostatic self-cleaning gecko-like adhesives. Journal of the Royal Society Interface, 15, 20170714.

108. Alizadehyazdi, V., \& Spenko, M. (2019). A microstructured adhesive gripper with piezoelectric controlled adhesion, cleaning, and sensing. Smart Materials and Structures, 28, 115037.

109. Alizadehyazdi, V., McQueney, E., Tanaka, K., \& Spenko, M. (2018). Ultrasonic and electrostatic self-cleaning microstructured adhesives for robotic grippers. Proceedings of the 2018 IEEE/RSJ International Conference on Intelligent Robots and Systems, Madrid, Spain, 7083-7088.

110. Alizadehyazdi, V., Bonthron, M., \& Spenko, M. (2020). Optimizing electrostatic cleaning for dust removal on gecko-inspired adhesives. Journal of Electrostatics, 108, 103499.

111. Autumn, K., Majidi, C., Groff, R. E., Dittmore, A., \& Fearing, R. (2006). Effective elastic modulus of isolated gecko setal arrays. Journal of Experimental Biology, 209, 3558-3568.

112. Bhushan, B. (2007). Adhesion of multi-level hierarchical attachment systems in gecko feet. Journal of Adhesion Science and Technology, 21, 1213-1258.

113. Gillies, A. G., Henry, A., Lin, H., Ren, A., Shiuan, K., Fearing, R. S., \& Full, R. J. (2014). Gecko toe and lamellar shear adhesion on macroscopic, engineered rough surfaces. Journal of Experimental Biology, 217, 283-289.

114. Kim, T. W., \& Bhushan, B. (2007). Adhesion analysis of multilevel hierarchical attachment system contacting with a rough surface. Journal of Adhesion Science and Technology, 21, 1-20.

115. Northen, M. T., \& Turner, K. L. (2006). Meso-scale adhesion testing of integrated micro- and nano-scale structures. Sensors and Actuators A, 130-131, 583-587.

116. Kustandi, T. S., Samper, V. D., Ng, W. S., Chong, A. S., \& Gao, H. (2007). Fabrication of a gecko-like hierarchical fibril array using a bonded porous alumina template. Journal of Micromechanics and Microengineering, 17, N75-N81.

117. Greiner, C., Arzt, E., \& Campo, A. D. (2009). Hierarchical gecko-like adhesives. Advanced Materials, 21, 479-482.
118. Lee, H., \& Bhushan, B. (2012). Fabrication and characterization of hierarchical nanostructured smart adhesion surfaces. Journal of Colloid and Interface Science, 372, 231-238.

119. Zhang, P., Liu, S. Y., \& Lv, H. (2013). Fabrication and properties of dual-level hierarchical structures mimicking gecko foot hairs. Journal of Nanoscience and Nanotechnology, 13, 781-786.

120. Rong, Z. X., Zhou, Y. M., Chen, B. G., Robertson, J., Federle, W., Hofmann, S., Steiner, U., \& Goldberg-Oppenheimer, P. (2014). Bio-inspired hierarchical polymer fiber-carbon nanotube adhesives. Advanced Materials, 26, 1456-1461.

121. Lee, J., Bush, B., Maboudian, R., \& Fearing, R. S. (2009). Gecko-inspired combined lamellar and nanofibrillar array for adhesion on nonplanar surface. Langmuir, 25, 12449-12453.

122. Asbeck, A., Dastoor, S., Parness, A., Fullerton, L., Esparza, N., Soto, D., Heyneman, B., \& Cutkosky, M. (2009). Climbing rough vertical surfaces with hierarchical directional adhesion. Proceedings of the 2009 IEEE International Conference on Robotics and Automation. Kobe, Japan, 2675-2680.

123. Murphy, M. P., Kim, S., \& Sitti, M. (2009). Enhanced adhesion by gecko-inspired hierarchical fibrillary adhesives. ACS Applied Materials \& Interfaces, 1, 849-855.

124. Krahn, J., \& Menon, C. (2012). Electro-dry-adhesion. Langmuir, $28,5438-5443$.

125. Krahn, J. M., Abraham, A. G. P., \& Menon, C. (2014). Polymeric electro-dry-adhesives for use on conducting surfaces. Proceedings of the Institution of Mechanical Engineers Part L-Journal of Materials-Design and Applications, 228, 109-114.

126. Iii, R. D., Parness, A., \& Spenko, M. (2014). Improving controllable adhesion on both rough and smooth surfaces with a hybrid electrostatic/gecko-like adhesive. Journal of the Royal Society Interface, 11, 20131089.

127. Dadkhah, M., Ruffatto, D., Zhao, Z. Y., \& Spenko, M. (2018). Increasing adhesion via a new electrode design and improved manufacturing in electrostatic/microstructured adhesives. Journal of Electrostatics, 91, 48-55.

128. Akherat, S. M. J. M., Karimi, M. A., Alizadehyazdi, V., Asalzadeh, S., \& Spenko, M. (2019). A tunable dielectric to improve electrostatic adhesion in electrostatic/microstructured adhesives. Journal of Electrostatics, 97, 58-70.

129. Chopra, V., Chudak, M., Hensel, R., Darhuber, A. A., \& Arzt, E. (2020). Enhancing dry adhesion of polymeric micropatterns by electric fields. ACS Applied Materials \& Interfaces, 12, 27708-27716.

130. Kim, S., Spenko, M., Trujillo, S., Heyneman, B., Santos, D., \& Cutkosky, M. R. (2008). Smooth vertical surface climbing with directional adhesion. IEEE Transactions on Robotics, 24, $65-74$.

131. Santos, D., Heyneman, B., Kim, S., Esparza, N., Cutkosky, M. R. (2008). Gecko-inspired climbing behaviors on vertical and overhanging surfaces. Proceedings of the 2008 IEEE International Conference on Robotics and Automation, Pasadena, USA, $1125-1131$

132. Hawkes, E. W., Ulmen, J., Esparza, N., Cutkosky, M. R. (2011). Scaling walls: applying dry adhesives to the Real World. Proceedings of the 2011 IEEE/RSJ International Conference on Intelligent Robots and Systems, San Francisco, USA, 5100-5106.

133. Hawkes, E. W., Eason, E. V., Asbeck, A. T., Asbeck, A. T., \& Cutkosky, M. R. (2013). The gecko's toe: Scaling directional adhesives for climbing applications. IEEE/ASME Transactions on Mechatronics, 18, 518-526.

134. Li, Y., Ahmed, A., Sameoto, D., \& Menon, C. (2011). Abigaille II: Toward the development of a spider-inspired climbing robot. Robotica, 30, 79-89.

135. Henrey, M., Ahmed, A., Boscariol, P., Shannon, L., \& Menon, C. (2014). Abigaille III: A versatile, bioinspired hexapod for 
scaling smooth vertical surfaces. Journal of Bionic Engineering, 11, 1-17.

136. Ko, H., Yi, H., \& Jeong, H. E. (2017). Wall and ceiling climbing quadruped robot with superior water repellency manufactured using 3D printing (UNIclimb). International Journal of Precision Engineering and Manufacturing-Green Technology, 4, 273-280.

137. Yu, Z. W., Shi, Y., Xie, J. X., Yang, S. X., \& Dai, Z. D. (2018). Design and analysis of a bionic adhesive foot for gecko robot climbing the ceiling. International Journal of Robotics and Automation, 33, 445-454.

138. Parness, A., Abcouwer, N., Fuller, C., Wiltsie, N., \& Kennedy, B. (2017). LEMUR 3: a limbed climbing robot for extreme terrain mobility in space. Proceedings of the 2017 IEEE International Conference on Robotics and Automation (ICRA), Singapore, 5467-5473.

139. Murphy, M. P., Kute, C., Menguc, Y., \& Sitti, M. (2011). Waalbot II: Adhesion recovery and improved performance of a climbing robot using fibrillary adhesives. International Journal of Robotics Research, 30, 118-133.

140. Krahn, J., Liu, Y., Sadeghi, A., \& Menon, C. (2011). A tailless timing belt climbing platform utilizing dry adhesives with mushroom caps. Smart Materials and Structures, 20, 115021.

141. Kalouche, S., Wiltsie, N., Su, H. J., \& Parness, A. (2014). Inchworm style gecko adhesive climbing robot. Proceedings of the 2014 IEEE/RSJ International Conference on Intelligent Robots and Systems, Chicago, USA, 2319-2324.

142. Hawkes, E. W., Christensen, D. L., \& Cutkosky, M. (2015). Vertical dry adhesive climbing with a 100x bodyweight payload. Proceedings of the 2015 IEEE International Conference on Robotics and Automation, Seattle, USA, 2015, 3762-3769.

143. Zhou, M., Tian, Y., Sameoto, D., Zhang, X. J., Meng, Y. G., \& Wen, S. Z. (2013). Controllable interfacial adhesion applied to transfer light and fragile objects by using gecko inspired mushroom-shaped pillar surface. ACS Applied Materials \& Interfaces, $5,10137-10144$.

144. Chu, Z. Y., Deng, J., Su, L., Cui, J., \& Sun, F. C. (2021). A geko-inspired adhesive robotic end effector for critical-contact manipulation. Science China Information Sciences. https://doi. org/10.1007/s11432-020-3152-7 (in press).

145. Dadkhah, M., Zhao, Z. Y., Wettels, N., \& Spenko, M. (2016). A self-aligning gripper using an electrostatic/gecko-like adhesive. Proceedings of the 2016 IEEE/RSJ International Conference on Intelligent Robots and Systems, Daejeon, Korea, 1006-1011.

146. Modabberifar, M., \& Spenko, M. (2018). A shape memory alloyactuated gecko-inspired robotic gripper. Sensors and Actuators A: Physical, 276, 76-82.

147. Modabberifar, M., \& Spenko, M. (2019). Development of a geckolike robotic gripper using Scott-Russell mechanisms. Robotica, 38, 541-549.

148. Song, S., \& Sitti, M. (2014). Soft gripper using micro-fibrillar adhesives for transfer printing. Advanced Materials, 26, 4901-4906.

149. Song, S., Majidi, C., \& Sitti, M. (2014). GeckoGripper: a soft, inflatable robotic gripper using gecko-inspired elastomer microfiber adhesives. Proceedings of the 2014 IEEE/RSJ International Conference on Intelligent Robots and Systems (IROS 2014), Chicago, USA, 4624-4629.

150. Song, S., Drotlef, D. M., Majidi, C., \& Sitti, M. (2017). Controllable load sharing for soft adhesive interfaces on three-dimensional surfaces. Proceedings of the National Academy of Sciences of the United States of America, 114, E4344-E4353.

151. Tian, H. M., Li, X. M., Shao, J. Y., Wang, C., Wang, Y., Tian, Y.Z., \& Liu, H. R. (2019). Gecko-effect inspired soft gripper with high and switchable adhesion for rough surfaces. Advanced Materials Interfaces, 6, 1900875.
152. Tian, H. M., Liu, H. R., Shao, J. Y., Li, S., Li, X. M., \& Chen, X. M. (2020). An electrically active gecko-effect soft gripper under a low voltage via mimicking gecko's adhesive structures and toe muscles. Soft Matter, 16, 5599-5608.

153. Parness, A., Heverly, M., Hilgemann, E., Copel, D., Wettels, N., Hilgendorf, T., White, V., \& Kennedy, B. (2013). On-OFF adhesive grippers for earth-orbit. Proceedings of the AIAA SPACE 2013 Conference and Exposition, San Diego, USA.

154. Jiang, H., Hawkes, E. W., Arutyunov, V., Tims, J., Cutkosky, M. R. (2015). Scaling controllable adhesives to grapple floating objects in space. Proceedings of the 2015 IEEE International Conference on Robotics and Automation (ICRA). Seattle, USA, 2828-2835.

155. Hawkes, E. W., Christensen, D. L., Han, A. K., Jiang, H., \& Cutkosky, M. R. (2015). Grasping without squeezing: shear adhesion gripper with fibrillary thin film. Proceedings of the 2015 IEEE International Conference on Robotics and Automation (ICRA), Seattle, USA, 2305-2312.

156. Hawkes, E. W., Jiang, H., Christensen, D. L., Han, A. K., \& Cutkosky, M. R. (2018). Grasping without squeezing: Design and modeling of shear-activated grippers. IEEE Transactions on Robotics, 34, 303-316.

157. Estrada, M. A., Hockman, B., Bylard, A., Hawkes, E. W., Cutkosky, M. R., \& Pavone, M. (2016). Free-flyer acquisition of spinning objects with gecko-inspired adhesives. Proceedings of the 2016 IEEE International Conference on Robotics and Automation (ICRA), Stockholm, Sweden, 4907-4913.

158. Estrada, M. A., Jiang, H., Noll, B., Hawkes, E. W., Cutkosky, M. R. (2017). Force and moment constraints of a curved surface gripper and wrist for assistive free flyers. Proceedings of the 2017 IEEE International Conference on Robotics and Automation (ICRA), Singapore, 2824-2830.

159. Hashizume, J., Huh, T. M., Suresh, S. A., \& Cutkosky, M. R. (2019). Capacitive sensing for a gripper with gecko-inspired adhesive film. IEEE Robotics and Automation Letters, 4, 677-683.

160. Jiang, H., Hawkes, E. W., Fuller, C. L., Estrada, M. A., Suresh, S. A., Abcouwer, N., Han, A. K., Wang, S. Q., Ploch, C. J., Parness, A., \& Cutkosky, M. (2017). A robotic device using gecko-inspired adhesives can grasp and manipulate large objects in microgravity. Science Robotics, 2017(2), eaan4545.

161. Hawkes, E. W., Christensen, D. L., Eason, E. V., Estrada, M. A., \& Cutkosky, M. R. (2013). Dynamic surface grasping with directional adhesion. Proceedings of the 2013 IEEE/RSJ International Conference on Intelligent Robots and Systems, Tokyo, Japan, 5487-5483.

162. Jiang, H., Pope, M. T., Hawkes, E. W., Christensen, D. L., Estrada, M. A., Parlier, A., Tran, R., \& Cutkosky, M R. (2014). Molding the dynamics of perching with opposed-grip mechanisms. Proceedings of the 2014 IEEE International Conference on Robotics and Automation (ICRA), Hong Kong, China, 3102-3108.

163. Hawkes, E. W., Jiang, H., \& Cutkosky, M. R. (2016). Three-dimensional dynamic surface grasping with dry adhesion. International Journal of Robotics Research, 35, 943-958.

164. Kalantari, A., Mahajan, K., Ruffatto, D., \& Spenko, M. (2015). Autonomous perching and take-off on vertical walls for a quadrotor micro air vehicle. Proceedings of the 2015 IEEE International Conference on Robotics and Automation (ICRA), Washington, USA, 4669-4674.

165. Cauligi, A., Chen, T. G., Suresh, S. A., Dille, M., Ruiz, R. G., Vargas, A. M., Pavone, M., \& Cutkosky, M. Design and development of a gecko-adhesive gripper for the Astrobee free-flying robot. Robotics. in submission. arXiv:2009.09151.

166. Glick, P., Suresh, S. A., Ruffatto, D., Cutkosky, M., Tolley, M. T., \& Parness, A. (2018). A soft robotic gripper with gecko-inspired adhesive. IEEE Robotics and Automation Letters, 3, 903-910.

167. Alizadehyazdi, V., Bonthron, M., \& Spenko, M. (2020). An electrostatic/gecko-inspired adhesives soft robotic gripper. IEEE Robotics and Automation Letters, 5, 4679-4686. 
168. Roberge, J. P., Ruotolo, W., Duchaine, V., \& Cutkosky, M. (2018). Improving industrial grippers with adhesion-controlled friction. IEEE Robotics and Automation Letters, 3, 1041-4048.

169. Mahdavi, A., Ferreira, L., Sundback, C., Nichol, J. W., Chan, E. P., Carte, D. J. D., Bettinger, C. J., Patanavanich, S., Chignozha, L., Ben-Joseph, E., Galakatos, A., Pryor, H., Irina, P., Masiakos Peter, T., William, F., Zumbuehl, A., Hong, S., Borenstein, J., Vacanti, J., ... Karp, J. M. (2008). A biodegradable and biocompatible geckoinspired tissue adhesive. Proceedings of the National Academy of Sciences of the United States of America, 105, 2307-2312.

170. Kwak, M. K., Jeong, H. E., \& Suh, K. Y. (2011). Rational design and enhanced biocompatibility of a dry adhesive medical skin patch. Advanced Materials, 23, 3949-3953.

171. Bae, W. G., Kim, D., Kwak, M. K., Ha, L., Kang, S. M., \& Suh, K. Y. (2012). Enhanced skin adhesive patch with modulus-tunable composite micropillars. Advanced Healthcare Materials, 2, 109-113.

172. Kim, T., Park, J., Sohn, J., Cho, D., \& Jeon, S. (2016). Bioinspired, highly stretchable, and conductive dry adhesive based on 1D-2D hybrid carbon nanocomposites for all-in-one ECG electrodes. ACS Nano, 10, 4770-4778.
173. Drotlef, D. M., Amjadi, M., Yunusa, M., \& Sitti, M. (2017). Bioinspired composite microfibers for skin adhesion and signal amplification of wearable sensors. Advanced Materials, 29, 1701353.

174. Irschick, D. J., Austin, C. C., Petren, K., Austin, C. C., Petren, K., Fisher, R. N., Losos, J. B., \& Ellers, O. (1996). A comparative analysis of clinging ability among pad-bearing lizards. Biological Journal of the Linnean Society, 59, 21-35.

175. Autumn, K. (2006). Properties, principles, and parameters of the gecko adhesive system. In A. M. Smith \& J. A. Callow (Eds.), Biological adhesives (pp. 225-256). Springer.

176. Wang, X., Yang, B. S., Tan, D., Li, Q., Song, B., Wu, Z. S., Campo, A. D., Kappl, M., Wang, Z. K., Gorb, S. N., Liu, S., \& Xue, L. J. (2020). Bioinspired footed soft robot with unidirectional all-terrain mobility. Materials Today, 35, 42-49.

Publisher's Note Springer Nature remains neutral with regard to jurisdictional claims in published maps and institutional affiliations. 